\title{
Internal Efficiency of Semiconductor Lasers With a Quantum-Confined Active Region
}

\author{
Levon V. Asryan, Serge Luryi, Fellow, IEEE, and Robert A. Suris
}

\begin{abstract}
We discuss in detail a new mechanism of nonlinearity of the light-current characteristic (LCC) in heterostructure lasers with reduced-dimensionality active regions, such as quantum wells (QWs), quantum wires (QWRs), and quantum dots (QDs). It arises from: 1) noninstantaneous carrier capture into the quantum-confined active region and 2) nonlinear (in the carrier density) recombination rate outside the active region. Because of 1 ), the carrier density outside the active region rises with injection current, even above threshold, and because of 2), the useful fraction of current (that ends up as output light) decreases. We derive a universal closed-form expression for the internal differential quantum efficiency $\eta_{\text {int }}$ that holds true for QD, QWR, and QW lasers. This expression directly relates the power and threshold characteristics. The key parameter, controlling $\eta_{\text {int }}$ and limiting both the output power and the LCC linearity, is the ratio of the threshold values of the recombination current outside the active region to the carrier capture current into the active region. Analysis of the LCC shape is shown to provide a method for revealing the dominant recombination channel outside the active region. A critical dependence of the power characteristics on the laser structure parameters is revealed. While the new mechanism and our formal expressions describing it are universal, we illustrate it by detailed exemplary calculations specific to QD lasers. These calculations suggest a clear path for improvement of their power characteristics. In properly optimized QD lasers, the LCC is linear and the internal quantum efficiency is close to unity up to very high injection-current densities $\left(15 \mathrm{kA} / \mathrm{cm}^{2}\right)$. Output powers in excess of $10 \mathrm{~W}$ at $\eta_{\text {int }}$ higher than $95 \%$ are shown to be attainable in broad-area devices. Our results indicate that QD lasers may possess an advantage for high-power applications.
\end{abstract}

Index Terms-Quantum dots (QDs), quantum wells (QWs), quantum wires (QWRs), semiconductor heterojunctions, semiconductor lasers.

\section{INTRODUCTION}

$\mathbf{R}$ EDUCING dimensionality of the active region improves significantly the performance of semiconductor lasers [1]. Quantum-well (QW) lasers have replaced bulk lasers in commercial applications [2], [3]. Further enhancement of device characteristics is expected for lasers with still lower dimensionality, such as quantum wire (QWR) and, especially, quantum dot

Manuscript received May 15, 2002; revised November 13, 2002. The work of L. V. Asryan and S. Luryi was supported by the AFOSR MURI under Grant F49620-00-1-0331 and by the New York State Center for Advanced Sensor Technology. The work of R. A. Suris was supported by the RFBR and by the Russian Program "Physics of Solid State Nanostructures."

L. V. Asryan is with the State University of New York at Stony Brook, Stony Brook, NY 11794-2350 USA, on leave from Ioffe Physico-Technical Institute, St. Petersburg 194021, Russia (e-mail: asryan@ece.sunysb.edu).

S. Luryi is with the State University of New York at Stony Brook, Stony Brook, NY 11794-2350 USA (e-mail: Serge.Luryi@ sunysb.edu).

R. A. Suris is with the Ioffe Physico-Technical Institute, St. Petersburg 194021, Russia (e-mail: suris@ theory.ioffe.rssi.ru).

Digital Object Identifier 10.1109/JQE.2002.808171
(QD) lasers [1], [4]. Recent research devoted to fabrication and improvement of QD lasers [5]-[15] indicates a number of potential advantages of such lasers over QW lasers in applications.

All reported QW, QWR, and QD laser structures have a common feature, which is the subject of interest in this paper. The quantum-confined active elements are embedded in a bulk reservoir region [which also serves as an optical confinement layer (OCL)] from where carriers are fed via some sort of a capture process. Since the capture process is never instantaneous, it gives rise to a current dependence of the carrier density in the reservoir, even above threshold when the carrier density in the active region itself is pinned by the steady-state generation condition. The increasing carrier density leads to an increase in the parasitic current corresponding to carrier recombination in the reservoir and contributes to a deviation of the internal differential quantum efficiency $\eta_{\text {int }}$ from unity. This fact was noted by a number of authors [16]-[23] but the actual reduction in $\eta_{\text {int }}$ due to this effect has never been quantified.

In this paper, we develop a quantitative theory of the "reservoir effect" under very general assumptions and obtain results that hold true for quantum-confined laser structures of all three dimensionalities. It turns out that this effect, combined with the nonlinear (superlinear in the carrier density) dependence of parasitic recombination rate in the reservoir, gives a major contribution to the nonlinearity (sublinearity) of the light-current characteristic (LCC) at high injection currents-comparable in magnitude to the entire experimentally observed LCC degradation. This suggests that the reservoir effect is one of the dominant mechanisms limiting both the output power and the linearity of the LCC. Neglecting other known mechanisms of nonlinearity, such as lattice and carrier heating, we derive a universal analytic expression for the internal quantum efficiency as a function of the injection-current density, valid for QD, QWR, and QW lasers. A critical dependence of the power characteristics of a laser with low-dimensional active region on the structure design is revealed, which suggests an improvement strategy. The general theory is complemented by detailed exemplary calculations specific to QD lasers.

The paper is organized as follows. In Section II, we present the rate equations underlying our analysis and outline the main assumptions and simplifications made, specializing to the case of a QD laser. Solutions of the rate equations for the confined carrier level occupancy in a QD, carrier density outside the active region, stimulated emission photon number, internal and external differential quantum efficiencies, and output power are obtained in Section III. Section IV analyzes these solutions for different limiting cases. Section V illustrates results of calculations for a specific QD laser structure. Section VI generalizes 
the results obtained for QD lasers to the case of QWR and QW lasers, bringing them into a universal form valid for all three types of confinement. Our conclusions are summarized in Section VII.

\section{Formulation of THE PROBLEM AND BASIC EQUATIONS}

Our calculation of the stimulated emission photon number and the output power is based on the steady-state rate equations. To neatly separate the subject for study and arrive at easy-to-analyze closed-form expressions, the following assumptions and simplifications are made.

\section{A. Main Assumptions}

1) Heating effects, which may cause sublinearity and roll-over of the LCC at high injection currents, and thus strongly limit the maximum output power in a semiconductor laser, are not treated here. Device degradation issues (such as catastrophic optical damage at the mirror facets at high-power densities [24], [25]) are not addressed either.

2) Spatial hole burning (SHB) and multilongitudinal behavior of the laser generation (due to a standing wave nature of the emitted light and spatially discrete arrangement of QDs [26]) are not taken into consideration. Hence the longitudinal $(z)$ coordinate dependence of the confined carrier level occupancies in QDs and of the free-carrier densities in the OCL is ignored

$$
f_{n}, f_{p}, n, p=\operatorname{const}(z)
$$

3) The free-carrier distribution in the transverse direction (direction of the structure growth, $x$ ) in the OCL is also assumed uniform

$$
n, p=\operatorname{const}(x) \text {. }
$$

This assumption means that the current is far from being controlled by diffusion across the OCL. Although transport to the active layer can affect the dynamic laser response [27], [28], it can be ignored in evaluating the steady-state output power.

4) The charge neutrality holds separately in the OCL and in the QDs, i.e., the free electron and hole densities are equal to each other and the electron and hole level occupancies (averaged over the QD ensemble) are also equal to each other

$$
n=p, \quad f_{n}=f_{p}
$$

This assumption reduces the total number of the rate equations by two- - only one equation is now required for free carriers and one for confined carriers.

5) The fraction of spontaneous emission in the lasing mode is ignored (see Section III-A).

6) The free-carrier density dependent component of the internal losses is ignored. This is justified for properly designed QD lasers, when parameters of the structure are well away from their critical values [26] and hence the carrier density is low. Thus, the internal losses are taken as constant, which is also supported by the experimental data [15]. This kind of internal losses may be attributed to the absorption outside the active region and the OCL (e.g., in the cladding layers), or to the scattering at rough surfaces [20].

7) Excited-state transitions in QDs are not taken into account.

Taking proper account of the above factors would tangibly complicate the calculations, while leaving our main results and conclusions unaffected. Their inclusion, to be treated in a separate study, can only lead to additional contributions to the fundamental nonlinearity mechanism studied here. This mechanism is inherent to any semiconductor laser based on a quantum-confined active region.

\section{B. Rate Equations}

With the above assumptions, we have the following set of rate equations for carriers confined in a $\mathrm{QD}$, free carriers in the OCL and photons:

$$
\begin{aligned}
\frac{\partial f_{n}}{\partial t}= & \sigma_{n} v_{n} n\left(1-f_{n}\right)-\sigma_{n} v_{n} n_{1} f_{n}-\frac{f_{n}^{2}}{\tau_{\mathrm{QD}}} \\
& -\frac{c}{\sqrt{\epsilon_{g}}} \frac{g^{\max }}{N_{S} S}\left(2 f_{n}-1\right) N \\
\frac{\partial n}{\partial t}= & \sigma_{n} v_{n} n_{1} \frac{N_{S}}{b} f_{n}-\sigma_{n} v_{n} n \frac{N_{S}}{b}\left(1-f_{n}\right) \\
& -B n^{2}+\frac{j}{e b} \\
\frac{\partial N}{\partial t}= & \frac{c}{\sqrt{\epsilon_{g}}} g^{\max }\left(2 f_{n}-1\right) N-\frac{c}{\sqrt{\epsilon_{g}}}\left(\beta+\alpha_{\mathrm{int}}\right) N
\end{aligned}
$$

where $\sigma_{n}$ is the cross-section of carrier capture into a QD, $v_{n}$ is the carrier thermal velocity, $\tau_{\mathrm{QD}}$ is the spontaneous radiative lifetime in a QD given by (8) in [29], $c$ is the light velocity in vacuum, $\sqrt{\epsilon_{g}}$ is the group index of the dispersive OCL material, $N_{S}$ is the surface density of QDs, $S=W L$ is the QD layer area (the cross-section of the junction), $W$ is the QD layer width (the lateral size of the device), $L$ is the QD layer length (the cavity length), $g^{\max }$ is the maximum (saturation) value of the modal gain spectrum peak (see [29] and (41) in [30]), $N$ is the number of photons in the lasing mode, $b$ is the OCL thickness, $B$ is the radiative constant for the OCL (given by (10) in [29]), $j$ is the injection-current density, $\beta=(1 / L) \ln (1 / R)$ is the mirror loss, $R$ is the facet reflectivity, and $\alpha_{\text {int }}$ is the modal internal loss [see assumption 6) above].

The quantity $n_{1}=N_{c}^{\mathrm{OCL}} \exp \left(-E_{n} / T\right)$, where $N_{c}^{\mathrm{OCL}}=$ $2\left(m_{c}^{\mathrm{OCL}} T / 2 \pi \hbar^{2}\right)^{3 / 2}, E_{n}$ is the carrier excitation energy from the QDs and the temperature $T$ is measured in units of energy.

Condition 4) of charge neutrality that holds separately in the OCL and in the QDs implies that the same capture cross section (denoted as $\sigma_{n}$ ) is assumed for both electrons and holes. If the capture cross sections are different, $\sigma_{n}$ should be regarded as an effective carrier capture cross section. For a large difference, the lower of the two capture cross sections will be effective, since the device performance will be controlled by the slower capture process. Thus, if the individual charge neutrality of QDs is not assumed, the following interpolation may be used for the effective capture cross section: $\sigma_{n} \sigma_{p} /\left(\sigma_{n}+\sigma_{p}\right)$. 
The first term on the right-hand side of (4) is the capture rate into a QD (in s ${ }^{-1}$ ). The second term is the rate of thermally excited escapes of carriers from a QD; it can be written as $f_{n} / \tau_{n}^{\text {esc }}$, where the characteristic time of escapes is [29]

$$
\tau_{n}^{\mathrm{esc}}=\frac{1}{\sigma_{n} v_{n} n_{1}} .
$$

For a specific structure considered in Section $\mathrm{V}, \tau_{n}^{\mathrm{esc}}=7 \mathrm{ps}$. The third and the fourth terms are the spontaneous and stimulated radiative recombination rates in a $\mathrm{QD}$, respectively; $N_{S} S$ is the number of QDs.

The maximum modal gain $g^{\max }$ is related to the maximum material gain $g_{0}^{\max }$ (gain reduced to one QD) as follows [29]:

$$
g^{\max }=N_{S} a^{2} \Gamma g_{0}^{\max }=\frac{1}{\mathcal{L}} N_{S} a^{3} g_{0}^{\max }
$$

where $a$ is the mean size of QDs, $\Gamma=a / \mathcal{L}$ is the optical confinement factor in a QD layer (along the transverse direction in the waveguide), and $\mathcal{L}$ is the characteristic length of the light confinement in the transverse direction in the waveguide. The factor

$$
N_{S} a^{2} \Gamma=\frac{N_{S} S a^{3}}{S \mathcal{L}}
$$

converting the material gain into the modal gain is simply the ratio of the total volume of all QDs (more precisely, the volume wherein carriers are confined) to the volume wherein a photon is confined.

With (8), the ratio $g^{\max } /\left(N_{S} S\right)$ in (4) becomes

$$
\frac{g^{\max }}{N_{S} S}=\frac{a^{3}}{S \mathcal{L}} g_{0}^{\max }=\frac{1}{S \mathcal{L}} \sigma_{\mathrm{QD}}^{\max }
$$

where $\sigma_{\mathrm{QD}}^{\max }=a^{3} g_{0}^{\max }$ is the maximum value of the stimulated emission cross-section in a QD averaged over the laser line (given by (22) in [29]). The factor $a^{3} /(S \mathcal{L})$ in (10) is the ratio of the volume wherein an electron is confined to the volume wherein a photon is confined. Since the emitted light penetrates into the cladding layers to some extent, $\mathcal{L}$ is larger than the OCL thickness $b$ and $S \mathcal{L}$ is larger than the OCL volume $V_{\mathrm{OCL}}=S b$. Actually, the electron wave function is also not completely localized within the QD, penetrating into the OCL and hence, $a$ in (8)-(10), is somewhat larger than the QD size. Nevertheless, since the material gain is inversely proportional to $a^{3}$ [29], the volume-wherein an electron is three-dimensionally (3-D) confined-drops out effectively of (10).

The first term on the right-hand side of (6) is the stimulated emission rate into the mode and the second term is the damping rate of the mode.

The first and the second terms on the right-hand side of (5) are the rates (in $\mathrm{cm}^{-3} \mathrm{~s}^{-1}$ ) of thermally excited escapes from QDs and capture into QDs, respectively. The third term is the spontaneous radiative recombination rate in the OCL and the last term is the rate of free-carrier injection into the OCL.

Since we neglect the carrier transport to QDs and assume uniform carrier distribution in the OCL, we effectively consider QDs as being evenly distributed over the OCL volume with a 3-D density of $N_{S} / b$ [cf. (5)].
We are interested in continuous-wave operation and hence use the steady-state $(\partial / \partial t=0)$ rate equations.

\section{MAin Results: Closed-Form Analytical SOlutions}

\section{A. Confined Carrier Level Occupancy in a $Q D$ (Carrier Density in the Active Region)}

From (6), it follows immediately that the confined carrier level occupancy above threshold pins at its threshold value

$$
f_{n}=\frac{1}{2}\left(1+\frac{\beta+\alpha_{\text {int }}}{g^{\max }}\right)=\frac{1}{2}\left(1+\frac{1}{\tau_{\mathrm{ph}} G^{\max }}\right)
$$

where we introduced the maximum modal gain in units of $\mathrm{s}^{-1}$ and the photon lifetime in the cavity as follows:

$$
\begin{aligned}
G^{\max } & =\frac{c}{\sqrt{\epsilon_{g}}} g^{\max } \\
\tau_{\mathrm{ph}} & =\frac{\sqrt{\epsilon_{g}}}{c} \frac{1}{\beta+\alpha_{\mathrm{int}}} .
\end{aligned}
$$

For zero-dimensional (0-D) active region (QDs), the level occupancy is an analog of the carrier density for a higher dimensional active region (QWR, QW, or bulk). The conclusion on the pinning of the carrier density above threshold is general and holds for bulk and quantum-confined active regions of all dimensionalities (QD, QWR, and QW), provided the above assumptions are justified. This is apparent from the steady-state rate equation for photons - if the spontaneous-emission fraction in the lasing mode and SHB are ignored, the modal gain spectrum peak pins above threshold: $g\left(n_{\text {act }}, p_{\text {act }}\right)=\beta+\alpha_{\text {int }}$, where $n_{\text {act }}$ and $p_{\text {act }}$ are the carrier densities in the active region. If, in addition, the charge neutrality holds in the active region ( $n_{\text {act }}=p_{\text {act }}$ ), a constant value of $n_{\text {act }}$ is obtained.

Taking account of the spontaneous emission fraction in the lasing mode causes the gain and hence $n_{\text {act }}$ to increase with the injection current, both approaching asymptotically their values obtained by neglecting this effect [31].

The SHB of the population inversion profile by the optical mode results in changes in the local carrier density in the active region [20]. Due to this effect, the average (over the longitudinal direction) modal gain and hence the level occupancy in a QD, are both higher than their threshold values [26]. The higher the injection current, the larger is this increase.

To our knowledge, no consideration has been given to charge neutrality violation in the active region of a semiconductor laser as it affects the output power. This effect may disrupt the pinning of the electron and hole level occupancies in a QD above threshold and cause their dependence on current, similarly to the way it causes their temperature dependence [30], [32]. We will address this issue in a separate work.

It should be emphasized that, for lasers based on a quantumconfined active region, only the carrier density within the active region is pinned above threshold. Outside the active region, the carrier density rises with the injection current. This point, at once apparent from the analysis below and of crucial importance, has been usually overlooked until recently even for much studied QW lasers. There have been reports of an increase in spontaneous emission from the OCL attributed to the increase in carrier density there with current above threshold 
[16]-[23]. There have also been calls [20] for studying the extent to which the absence of carrier density pinning outside the active region contributes to the reduction of the internal differential quantum efficiency $\eta_{\text {int }}$. However, to our knowledge, no quantitative studies of this effect have been reported.

The approach used below is general and based directly on the rate equations. The following analysis is performed for a specific case of a QD laser and then is generalized for a semiconductor laser based on quantum-confined active region of an arbitrary dimensionality (Section VI). Hence, all our conclusions apply equally to QD, QWR, and QW lasers.

\section{B. Free-Carrier Density in the OCL and Photon Number}

Substituting $f_{n}$ from (11) into (4) and (5) gives a set of equations for $n$ and $N$ versus injection current. From (4), we have

$$
n=n_{\text {th }}\left(1+\frac{j_{\text {stim }}}{j_{\text {capt }, \text { th }}}\right)
$$

where $n_{\text {th }}$ is the carrier density in the OCL at the lasing threshold (not to be confused with the threshold carrier density in the active region) given by

$$
n_{\mathrm{th}}=n_{1} \frac{f_{n}}{1-f_{n}}+\frac{1}{\sigma_{n} v_{n} \tau_{\mathrm{QD}}} \frac{f_{n}^{2}}{1-f_{n}} .
$$

The stimulated recombination current density is

$$
j_{\mathrm{stim}}=e \frac{1}{S} \frac{N}{\tau_{\mathrm{ph}}} .
$$

The current density of the carrier capture into the QD ensemble at the lasing threshold [see the second term on the right-hand side of (5)] is

$$
\begin{aligned}
j_{\text {capt,th }} & =e b n_{\text {th }}\left[\sigma_{n} v_{n} \frac{N_{S}}{b}\left(1-f_{n}\right)\right] \\
& =e\left[\sigma_{n} v_{n} N_{S}\left(1-f_{n}\right)\right] n_{\mathrm{th}} .
\end{aligned}
$$

The terms in brackets may be regarded as the reciprocal of the "capture time" and the "capture velocity" into the QD ensemble (see Section III-F1 and Section VI).

With (4), we write (5) as follows

$$
j=j_{\mathrm{th}}+e b B\left(n^{2}-n_{\mathrm{th}}^{2}\right)+j_{\mathrm{stim}}
$$

where $j_{\text {th }}$ is the threshold current density given by

$$
j_{\mathrm{th}}=e N_{S} \frac{f_{n}^{2}}{\tau_{\mathrm{QD}}}+e b B n_{\mathrm{th}}^{2} .
$$

The second terms on the right-hand sides of (14) and (18) represent, respectively, the increase of the carrier density and of the spontaneous radiative recombination current density in the OCL over their threshold values, caused by the excess of the injection-current density above threshold.

Inserting $n$ from (14) into (18) yields a quadratic equation in $j_{\text {stim }}$ (i.e., in the photon number $N$ )

$$
\frac{j-j_{\text {th }}}{j_{\mathrm{th}}^{\mathrm{OCL}}}=\left(1+\frac{j_{\mathrm{stim}}}{j_{\text {capt,th }}}\right)^{2}-1+\frac{j_{\mathrm{stim}}}{j_{\mathrm{th}}^{\mathrm{OCL}}}
$$

where $j_{\mathrm{th}}^{\mathrm{OCL}}$ is the threshold current density component corresponding to the spontaneous recombination in the OCL [the second term on the right-hand side of (19)]

$$
j_{\mathrm{th}}^{\mathrm{OCL}}=e b B n_{\mathrm{th}}^{2} .
$$

Note that $j_{\text {capt,th }}$ is not a component of the threshold current density. As can be seen from (4) and (5)

$$
j_{\text {capt }, \text { th }}-j_{\text {esc }}=j_{\text {spon }}^{\mathrm{QD}}
$$

where $j_{\mathrm{esc}}$ is the current density of carrier escape from the QD ensemble and $j_{\text {spon }}^{\mathrm{QD}}$ is the threshold current density component corresponding to the spontaneous recombination in QDs, viz.

$$
\begin{aligned}
j_{\mathrm{esc}} & =e N_{S} f_{n}\left(\sigma_{n} v_{n} n_{1}\right)=e N_{S} \frac{f_{n}}{\tau_{\mathrm{esc}}} \\
j_{\mathrm{Spon}}^{\mathrm{QD}} & =e N_{S} \frac{f_{n}^{2}}{\tau_{\mathrm{QD}}} .
\end{aligned}
$$

Since $f_{n}$ pins at its threshold value above threshold, so do $j_{\text {esc }}$ and $j_{\text {spon }}^{\mathrm{QD}}$.

Thus, the quadratic (in $j_{\text {stim }}$ and hence in the photon number $N$ ) term enters into (20) for $j-j_{\text {th }}$. This stems from the bimolecular (quadratic in carrier density) character of the recombination in the OCL [see the second term on the right-hand side of (18)] and from the linearity of $n$ with $N$ [see the second term on the right-hand side of (14)]. Solution of (20) gives $j_{\text {stim }}$ (i.e., $N$ ) as a function of $j-j_{\text {th }}$; substituting this function into (14) we obtain an expression for $n$. It is natural and convenient to express these quantities in terms of the internal differential quantum efficiency $\eta_{\text {int }}$. Thus, we obtain the following equations for the surface density of photons (the photon number per unit area of the active layer) and carrier density in the OCL:

$$
\begin{aligned}
\frac{N}{S} & =\frac{j-j_{\text {th }}}{e} \eta_{\text {int }} \tau_{\mathrm{ph}} \\
n & =n_{\text {th }}\left(1+\frac{j-j_{\text {th }}}{j_{\text {capt }, \text { th }}} \eta_{\text {int }}\right)
\end{aligned}
$$

where $\eta_{\text {int }}$ itself depends on $j-j_{\text {th }}$.

When the nonradiative Auger recombination in the OCL is also taken into account in addition to the spontaneous radiative recombination, instead of (18) we have

$$
j-j_{\mathrm{th}}=e b B\left(n^{2}-n_{\mathrm{th}}^{2}\right)+e b C\left(n^{3}-n_{\mathrm{th}}^{3}\right)+j_{\mathrm{stim}}
$$

with the threshold current density being given as [compare with (19)]

$$
j_{\mathrm{th}}=e N_{S} \frac{f_{n}^{2}}{\tau_{\mathrm{QD}}}+e b B n_{\mathrm{th}}^{2}+e b C n_{\mathrm{th}}^{3}
$$

where $C$ is the Auger recombination constant in the OCL (in $\mathrm{cm}^{6} / \mathrm{s}$ ). Inserting $n$ from (14) into (27) gives a cubic equation in $j_{\text {stim }}$

$$
\begin{aligned}
j-j_{\text {th }}=e b C n_{\text {th }}^{3}\left[\left(1+\frac{j_{\text {stim }}}{j_{\text {capt th }}}\right)^{3}-1\right] \\
+e b B n_{\text {th }}^{2}\left[\left(1+\frac{j_{\text {stim }}}{j_{\text {capt th }}}\right)^{2}-1\right]+j_{\text {stim }} .
\end{aligned}
$$




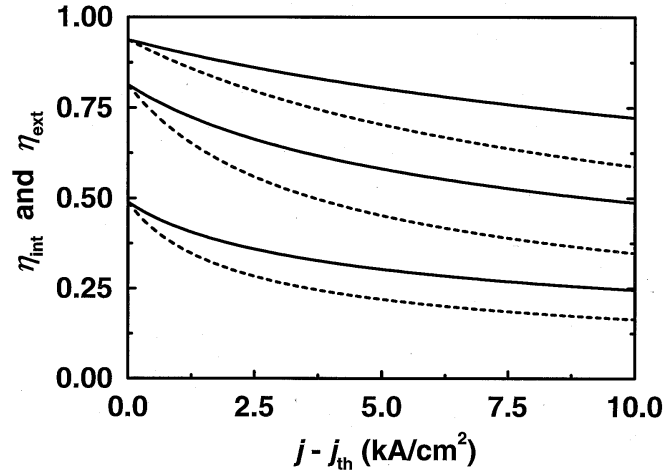

Fig. 1. Internal quantum efficiency (solid curves) and external differential efficiency (dashed curves) of a QD laser against excess injection-current density for different ratios $j_{\mathrm{th}}^{\mathrm{OCL}} / j_{\text {capt,th }}$. The values of $j_{\mathrm{th}}^{\mathrm{OCL}} / j_{\text {capt,th }}$ for the different curves (from the top down) are $0.033,0.115$, and 0.523 . The $j_{\text {th }}$ values are $31.54,83.85$, and $336.99 \mathrm{~A} / \mathrm{cm}^{2}$; they correspond to $N_{S}$ values of $4 \times, 3.3 \times$, and $2.9 \times 10^{10} \mathrm{~cm}^{-2}$, respectively.

The cubic term in (29) is due to Auger recombination. A closed-form solution of (29) in the general case of an arbitrary $j-j_{\text {th }}$, obtained using Cardano's formula, will not be presented here for reasons of space.

The following analysis is based on (20). We will turn to (29) in Section IV-B2.

\section{Internal Differential Quantum Efficiency}

Above the lasing threshold, the internal differential quantum efficiency of a semiconductor laser is defined as the fraction of the excess of the injection current over the threshold current that results in stimulated emission

$$
\eta_{\text {int }}=\frac{j_{\text {stim }}}{j-j_{\mathrm{th}}}=\frac{e \frac{N}{\tau_{\mathrm{ph}}}}{I-I_{\mathrm{th}}}
$$

where $I=S j$ and $I_{\mathrm{th}}=S j_{\mathrm{th}}$ are the injection and threshold currents, respectively. Solution of (20) yields

$$
\eta_{\text {int }}=\frac{1}{\frac{1}{2}+\frac{j_{\text {th }}^{\text {OCL }}}{j_{\text {capt }, \text { h }}}+\sqrt{\left(\frac{1}{2}+\frac{j_{\text {th }}^{\text {OCL }}}{j_{\text {capt th }}}\right)^{2}+\frac{j_{\text {th }}^{\text {OCL }}}{j_{\text {capt th }}} \frac{j-j_{\text {th }}}{j_{\text {capt th }}}}} .
$$

Thus, $\eta_{\text {int }}$ is seen to be a decreasing function of $j-j_{\text {th }}$ (Fig. 1).

Since the level occupancy in a QD (the carrier density in the higher dimensional active region-QWR or QW) clamps above threshold, so do the nonradiative (if any) and the spontaneous radiative recombination currents in QDs (in the active region), being controlled by this level occupancy (by the carrier density in the active region). For this reason, the increase (over the threshold current) in the fraction of the injection current delivered to the active region goes entirely into the stimulated recombination: $\Delta j_{\text {act }}=j_{\text {stim }}$. Hence, above threshold, $\eta_{\text {int }}$ is equal to the differential injection efficiency $\eta_{\text {inj }}$ defined as the fraction of the injection-current excess that enters the active region $\Delta j_{\text {act }} /\left(j-j_{\text {th }}\right)$.

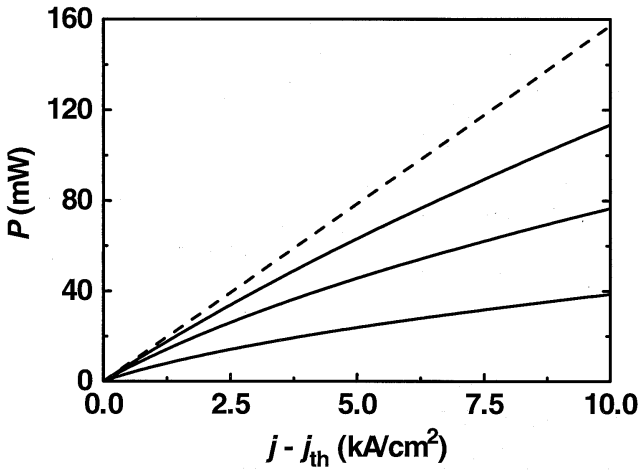

Fig. 2. Light-current characteristics of a QD laser for different ratios

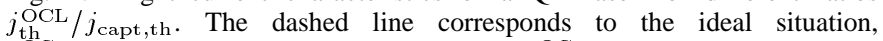
$j_{\mathrm{th}}^{\mathrm{OCL}} / j_{\text {capt,th }}=0\left(\eta_{\text {int }}=1\right)$. The values of $j_{\mathrm{th}}^{\mathrm{OCL}} / j_{\text {capt th }}, j_{\text {th }}$ and $N_{S}$ are the same as in Fig. 1.

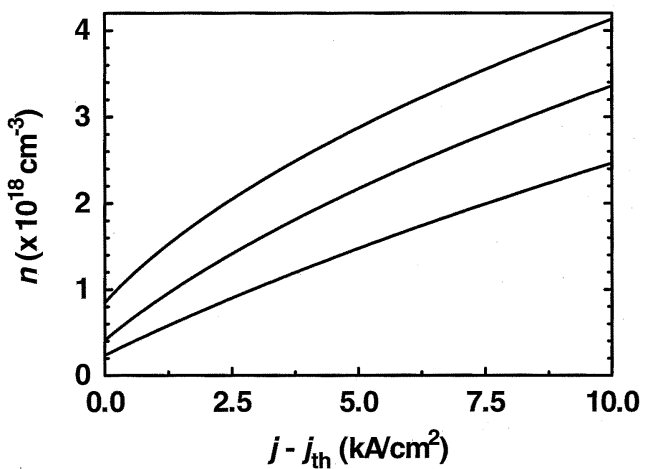

Fig. 3. Injection-current density dependence of the free-carrier density in the OCL of a QD laser for different ratios $j_{\mathrm{th}}^{\mathrm{OCL}} / j_{\text {capt,th. The values of }}$ $j_{\text {th }}^{\text {OCL }} / j_{\text {capt }, \text { th }}, j_{\text {th }}$ and $N_{S}$ (from bottom to top) are the same as in Fig. 1.

\section{Output Power Versus Injection Current (Light-Current Characteristic)}

The output optical power is of the form

$$
P=\hbar \omega \frac{N}{\tau_{\mathrm{ph}}} \eta_{\mathrm{opt}}=\frac{\hbar \omega}{e} I_{\mathrm{stim}} \eta_{\mathrm{opt}}=\frac{\hbar \omega}{e}\left(I-I_{\mathrm{th}}\right) \eta_{\mathrm{int}} \eta_{\mathrm{opt}}
$$

where $\hbar \omega$ is the photon energy, $I_{\text {stim }}=S j_{\text {stim }}$ is the stimulated recombination current, and

$$
\eta_{\mathrm{opt}}=\frac{\beta}{\beta+\alpha_{\mathrm{int}}}
$$

is the optical efficiency of the laser cavity for light emitted from both edges (Fabry-Perot cavity is considered here); $\eta_{\text {opt }}$ accounts for the fact that a quantity of photons disappears through internal losses, rather than leaves the cavity through the mirrors to be collected as useful power. The dependence of $\eta_{\text {int }}$ on the injection-current is given by (31).

It is evident from (31) and (32) that the dependence of the output power on the injection current is sublinear (see Fig. 2 and Section IV-B and Section IV-C below for more details). This sublinearity of the LCC stems from the absence of pinning of the carrier density $n$ outside the active region (Fig. 3) and from the superlinearity of the recombination rate with respect to $n$ in that region.

Equation (14), relating $n$ to $N$ by a linear dependence, is unaffected by a specific type of the dominant recombination channel in the OCL. For a monomolecular recombination (linear in $n$, 


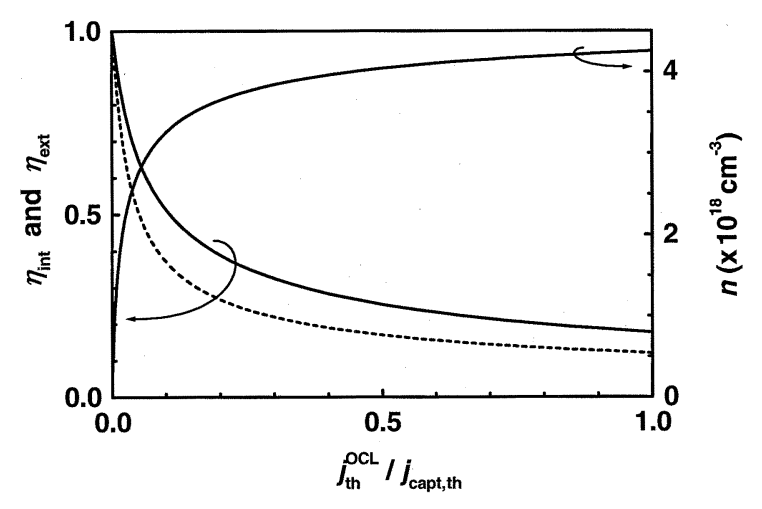

Fig. 4. Internal quantum efficiency (solid curve), external differential efficiency (dashed curve), and free-carrier density in the OCL (right axis) of a QD laser against ratio $j_{\mathrm{th}}^{\mathrm{OCL}} / j_{\text {capt,th}}$. Injection-current density $j=10 \mathrm{kA} / \mathrm{cm}^{2}$; the variation of $j_{\mathrm{th}}^{\mathrm{OCL}} / j_{\text {capt,th }}$ in the range shown (from $8 \times 10^{-4}$ to 1 ) is accomplished by changing $N_{S}$ from $20 \times$ to $2.81 \times 10^{10} \mathrm{~cm}^{-2}$.

such as recombination on impurities), this yields a linear dependence of $j-j_{\text {th }}$ on $N$ instead of (20). If this were the dominant channel, the LCC would be linear at any $j-j_{\text {th }}$ and the internal quantum efficiency would then be constant, albeit less than unity

$$
\eta_{\mathrm{int}}=\frac{1}{1+\frac{1}{\tau_{\mathrm{nr}}} \frac{1}{\sigma_{n} v_{n} \frac{N_{S}}{b}\left(1-f_{n}\right)}}=\frac{1}{1+\frac{\tau_{\mathrm{capt}}}{\tau_{\mathrm{nr}}}}
$$

where $\tau_{\mathrm{nr}}$ is the nonradiative recombination lifetime and $\tau_{\text {capt }}$ is the "capture time" into the QD ensemble (see Section III-F1). It should be pointed out, however, that recombination via nonradiative centers is never the dominant channel in high-quality laser structures where the density of such centers is low.

\section{E. External Differential Quantum Efficiency}

The external differential quantum efficiency is defined as

$$
\eta_{\mathrm{ext}}=\frac{e}{\hbar \omega} \frac{\partial P}{\partial I}=\frac{e}{\tau_{\mathrm{ph}}} \frac{\partial N}{\partial I} \eta_{\mathrm{opt}}
$$

In view of the current-dependence of the internal quantum efficiency (Fig. 1), the product of the latter and the optical efficiency $\eta_{\text {opt }}$ does not present in general the external efficiency. From (32), we get

$$
\eta_{\text {ext }}=\left[1+\frac{\partial \ln \eta_{\text {int }}}{\partial \ln \left(I-I_{\text {th }}\right)}\right] \eta_{\text {int }} \eta_{\text {opt }} .
$$

Since $\eta_{\text {int }}$ is a decreasing function of $I-I_{\mathrm{th}}$, the expression in the brackets is less than unity. Hence, at $I>I_{\mathrm{th}}$, due to the LCC sublinearity, $\eta_{\text {ext }}<\eta_{\text {int }}$ even for $\eta_{\mathrm{opt}}=1$ (Figs. 1 and 4).

With (31), the following equation for $\eta_{\text {ext }}$ is obtained:

$$
\begin{aligned}
& \eta_{\text {ext }}=\left[1-\frac{1}{2} \frac{\frac{j_{\text {th }}^{\text {OCL }}}{j_{\text {capt,th }}} \frac{j-j_{\text {th }}}{j_{\text {capt th }}}}{\sqrt{\left(\frac{1}{2}+\frac{j_{\text {th }}^{\text {OCL }}}{j_{\text {capt th }}}\right)^{2}+\frac{j_{\text {th }}^{\text {OCL }}}{j_{\text {capt th }}} \frac{j-j_{\text {th }}}{j_{\text {capt th }}}}} \eta_{\text {int }}\right] \\
& \times \eta_{\text {int }} \eta_{\text {opt }} .
\end{aligned}
$$

\section{F. Key Parameter Controlling Power Characteristics}

As seen from (25), (26), (31), (32), and (37), for a given $j-$ $j_{\text {th }}$, the internal and external quantum efficiencies, the free-car-

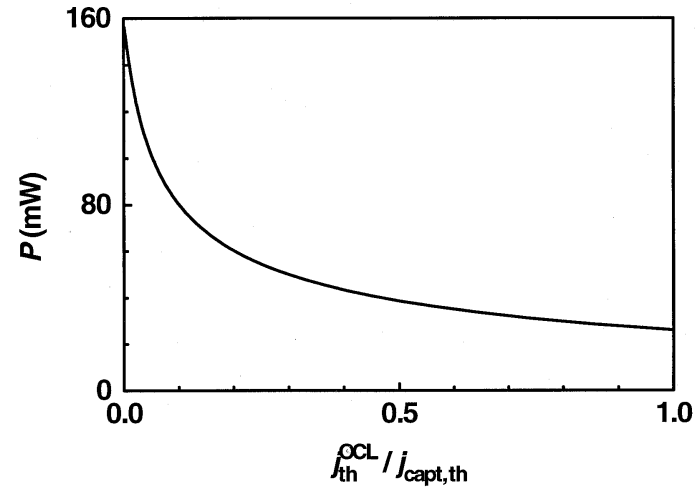

Fig. 5. Output power of a QD laser against ratio $j_{\mathrm{th}}^{\mathrm{OCL}} / j_{\text {capt, th }}$. The injection-current density and parameters are the same as in Fig. 4.

rier density in the OCL, the stimulated photon number and the output power are all controlled by the ratio

$$
\frac{j_{\text {th }}^{\mathrm{OCL}}}{j_{\mathrm{capt}, \mathrm{th}}}=\frac{B n_{\mathrm{th}}}{\sigma_{n} v_{n} \frac{N_{S}}{b}\left(1-f_{n}\right)} .
$$

It depends critically on the structure parameters (see below). The lower the ratio (38), the closer is $\eta_{\text {int }}$ to unity (Figs. 1 and 4) and the more linear is the LCC (Fig. 2). Ideally, when this ratio vanishes (e.g., when $j_{\mathrm{th}}^{\mathrm{OCL}}=0$-no recombination in the $\mathrm{OCL}$ ), $\eta_{\text {int }}=1$ at an arbitrary injection current and the LCC is linear. In this case, $N, n$ and $P$ [given by (25), (26) and (32), wherein $\left.\eta_{\text {int }}=1\right]$ are linear functions of $j-j_{\mathrm{th}}$. In general, however, $j_{\mathrm{th}}^{\mathrm{OCL}}$ is a tangible fraction of the total $j_{\mathrm{th}}$. It is this component that should be first of all suppressed to minimize $j_{\text {th }}$ and optimize the structure [26], [29], [30], [32]-[34]. The conclusion that high-power performance of a laser is inseparably controlled by the threshold characteristics is of great importance. The higher the excess of the injection current over the threshold current, the stronger this relation is manifested (Figs. 1 and 2). The higher the required output power is, the lower should be $j_{\text {th }}$ (Figs. 2 and 5). Although we have reached this conclusion in the instance of a QD laser, it will be shown in Section VI that all the above equations-and hence the conclusion-remain valid for lasers with a quantum-confined active region of arbitrary dimensionality. Therefore, the usual approach to obtaining high powers in injection lasers, which neglects or considers separately the issue of reducing $j_{\text {th }}$, appears to be rather off-base. We remark, finally, that since QD lasers offer the lowest threshold current density among all currently used semiconductor lasers, our results prove their another-extremely important-potential advantage, namely the possibility of achieving the highest output powers (see Section V).

1) “Capture Time" Into the QD Ensemble: The ratio in (38) can be written as follows:

$$
\frac{j_{\mathrm{th}}^{\mathrm{OCL}}}{j_{\text {capt,th }}}=\frac{\tau_{\text {capt }}}{\tau_{\text {th }}^{\mathrm{OCL}}}
$$

where the time constants

$$
\begin{aligned}
\tau_{\mathrm{th}}^{\mathrm{OCL}} & =\frac{1}{B n_{\mathrm{th}}} \\
\tau_{\text {capt }} & =\frac{1}{\sigma_{n} v_{n} \frac{N_{S}}{b}\left(1-f_{n}\right)}=\frac{\tau_{\text {capt }, 0}}{1-f_{n}}
\end{aligned}
$$


may be regarded, respectively, as the recombination time in the OCL at the lasing threshold and the "capture time" into the QD ensemble. The time constant

$$
\tau_{\text {capt }, 0}=\frac{1}{\sigma_{n} v_{n} \frac{N_{S}}{b}}
$$

has the meaning of a "capture time" into the unoccupied QD ensemble (when $f_{n}=0$ ). For a specific structure considered in Section $\mathrm{V}, \tau_{\text {capt }, 0}=97 \mathrm{ps}$.

With (41), the stimulated emission current density and the output power become

$$
\begin{aligned}
j_{\text {stim }} & =\frac{e b\left(n-n_{\mathrm{th}}\right)}{\tau_{\text {capt }}}=\frac{e b\left(n-n_{\mathrm{th}}\right)\left(1-f_{n}\right)}{\tau_{\text {capt }, 0}} \\
P & =\hbar \omega \frac{V_{\mathrm{OCL}}\left(n-n_{\mathrm{th}}\right)}{\tau_{\text {capt }}} \eta_{\mathrm{opt}} \\
& =\hbar \omega \frac{V_{\mathrm{OCL}}\left(n-n_{\mathrm{th}}\right)\left(1-f_{n}\right)}{\tau_{\text {capt }, 0}} \eta_{\mathrm{opt}} .
\end{aligned}
$$

Equations (43) and (44) have an evident meaning: the carrier supply to QDs, the origin of all stimulated photons and optical power, occurs by the capture process (see discussions in Section IV-B and Section IV-C).

For both nonlinear recombination channels in the OCL, either spontaneous radiation (presently considered case), or nonradiative Auger recombination (considered below), the time $\tau_{\text {th }}^{\mathrm{OCL}}$ depends on the carrier density in the OCL at threshold, as $\tau_{\text {th }}^{\text {OCL }} \propto 1 / n_{\text {th }}$ or $1 / n_{\text {th }}^{2}$, respectively. The "capture time" $\tau_{\text {capt }}$ is inversely proportional to the 3-D density of unoccupied states in the QD ensemble $\left(N_{S} / b\right)\left(1-f_{n}\right)$. Both $f_{n}$ and $n_{\text {th }}$ [see (11) and (15) ] depend crucially on the structure parameters. For this reason, $\tau_{\mathrm{th}}^{\mathrm{OCL}}$ and $\tau_{\text {capt }}$ thus defined are not the true time constants describing the respective processes [in contrast to $\tau_{\mathrm{nr}}$, which is independent of the carrier density-see (34)]. Further still, $\tau_{\text {capt }}$ is a characteristic of the entire QD ensemble, rather than of a single QD. In the strict sense, the time for the carrier capture into a single QD can not be introduced properly (though this is sometimes done in the literature). An adequate physical quantity, describing correctly the carrier capture into a single $\mathrm{QD}$, is the capture cross-section $\sigma_{n}$. For the reasons above and to avoid misinterpreting, we will not use $\tau_{\mathrm{th}}^{\mathrm{OCL}}$ and $\tau_{\text {capt }}$ frequently.

2) "Critical" Dependence on the Structure Design: With (15), (38) can be written as

$$
\frac{j_{\mathrm{th}}^{\mathrm{OCL}}}{j_{\text {capt,th }}}=\frac{B}{\sigma_{n} v_{n} \frac{N_{S}}{b}}\left[n_{1}+\frac{1}{\sigma_{n} v_{n} \tau_{\mathrm{QD}}} f_{n}\right] \frac{f_{n}}{\left(1-f_{n}\right)^{2}} .
$$

The level occupancy in a QD (11) ranges within $1 / 2<f_{n}<$ 1 . The low value of $1 / 2$ corresponds to vanishing total losses (infinitely long cavities and zero internal losses), when the lasing threshold is close to the transparency (inversion) threshold. The high value of 1 is for the highest tolerable losses determined by the maximum modal gain $g^{\max }$ (equivalently, when one of the structure parameters $N_{S}, \delta$, or $L$, is close to its critical tolerable value, $N_{S}^{\min }, \delta^{\max }$, or $L^{\min }$, respectively [26]).

The minimum value of the ratio $j_{\mathrm{th}}^{\mathrm{OCL}} / j_{\text {capt,th }}$ (obtained when $\beta+\alpha_{\text {int }}=0$, and hence $f_{n}=1 / 2$ ) is typically much less than unity. Thus, for a specific structure considered in Section $\mathrm{V}$, it is $10^{-3}$ at room temperature.
When $f_{n} \rightarrow 1, n_{\text {th }}$ increases as $1 /\left(1-f_{n}\right)$-see (15). Hence, $j_{\mathrm{th}}^{\mathrm{OCL}}$ increases as $1 /\left(1-f_{n}\right)^{2}$ [so does the total $j_{\mathrm{th}}$-see (19) and (21)]. As this takes place, the capture current density tends to a constant value [this is seen from (17) and (15), or, equivalently, from (22)-(24)]

$$
\left.j_{\text {capt }, \mathrm{th}}\right|_{f_{n} \rightarrow 1}=e N_{S}\left(\frac{1}{\tau_{\mathrm{esc}}}+\frac{1}{\tau_{\mathrm{QD}}}\right) .
$$

Hence, $j_{\mathrm{th}}^{\mathrm{OCL}} / j_{\text {capt,th }} \rightarrow \infty$. As a result, the internal quantum efficiency and the output power drop to zero (Figs. 4 and 5).

In view of the critical dependence of the ratio $j_{\text {th }}^{\mathrm{OCL}} / j_{\text {capt,th }}$ on structure parameters, optimization of the design is of crucial importance. Thus, for the optimized structure of Section V $\left(f_{n}^{\text {opt }}=0.655\right)$, this ratio is $3 \times 10^{-3}$, i.e., it is close to its minimum value. What this means is the LCC of such an optimized structure will be linear up to very high injection currents (see Section V).

The critical dependence of threshold and power characteristics on the structure design, stemming from such behavior of $n_{\text {th }}$, is also inherent in QWR and QW lasers (see Section VI).

\section{ANALYSiS of SOLUtions FOR DifFERENT Limiting CASES}

We shall consider different situations and limiting cases separately.

\section{A. Linear Portion of the LCC (Low Injection Currents)}

The injection-current density is slightly increased over $j_{\mathrm{th}}$. In the linear approximation, the quantities $N, n$ and $P$ are given by (25), (26), and (32), respectively, by substituting therein the value of $\eta_{\text {int }}$ at $j=j_{\text {th }}$

$$
\left.\eta_{\mathrm{int}}\right|_{j=j_{\mathrm{th}}}=\frac{1}{1+2 \frac{j_{\mathrm{th}}^{\mathrm{OCL}}}{j_{\mathrm{capt} \text { th }}}} .
$$

The internal quantum efficiency is less than unity even at $j=$ $j_{\text {th }}$. When one of the structure parameters $\left(N_{S}, \delta\right.$, or $\left.L\right)$ is close to its critical tolerable value $\left(N_{S}^{\min }, \delta^{\max }\right.$, or $L^{\mathrm{min}}$, respectively), $\left.\eta_{\mathrm{int}}\right|_{j=j_{\mathrm{th}}} \rightarrow 0$ and the linear portion of the LCC disappears.

\section{B. Nonlinear Portion of the LCC (High Injection Currents)—Capture-Limited Performance}

1) Asymptotic Equations for the Case When the Dominant Recombination Channel in the OCL is Spontaneous Radiative Recombination: At high injection currents, when

$$
\frac{j-j_{\mathrm{th}}}{j_{\mathrm{capt}, \mathrm{th}}} \gg \frac{j_{\text {capt,th }}}{j_{\mathrm{th}}^{\mathrm{OCL}}}\left(\frac{1}{2}+\frac{j_{\mathrm{th}}^{\mathrm{OCL}}}{j_{\mathrm{capt}, \mathrm{th}}}\right)^{2}
$$

(31), (26), (32), and (37) read as

$$
\begin{aligned}
\eta_{\text {int }} & =\frac{j_{\text {capt }, \text { th }}}{\sqrt{j_{\mathrm{th}}^{\mathrm{OCL}}\left(j-j_{\mathrm{th}}\right)}}=\frac{\sigma_{n} v_{n} N_{S}\left(1-f_{n}\right)}{\sqrt{b B \frac{j-j_{\mathrm{th}}}{e}}} \\
n & =n_{\mathrm{th}} \sqrt{\frac{j-j_{\mathrm{th}}}{j_{\mathrm{th}}^{\mathrm{OCL}}}}=\sqrt{\frac{j-j_{\mathrm{th}}}{e b B}}
\end{aligned}
$$




$$
\begin{aligned}
P & =\frac{\hbar \omega}{e} S j_{\mathrm{capt}, \mathrm{th}} \sqrt{\frac{j-j_{\mathrm{th}}}{j_{\mathrm{th}}^{\mathrm{OCL}}}} \eta_{\mathrm{opt}} \\
& =\hbar \omega\left(N_{S} S\right) \sigma_{n} v_{n}\left(1-f_{n}\right) \sqrt{\frac{j-j_{\mathrm{th}}}{e b B}} \eta_{\mathrm{opt}} \\
\eta_{\mathrm{ext}} & =\frac{1}{2} \eta_{\mathrm{int}} \eta_{\mathrm{opt}} \cdot
\end{aligned}
$$

In view of (48), $\eta_{\text {int }} \ll 1$ and $n \gg n_{\text {th }}$. Because of the rapid decrease of $\eta_{\text {int }}$ with $j-j_{\text {th }}$, the ratio $\eta_{\text {ext }} / \eta_{\text {opt }}$ reduces to one half of $\eta_{\text {int }}$; note that (52) readily obtains from (36) by taking $\eta_{\text {int }} \propto 1 / \sqrt{j-j_{\text {th }}}$. form

With (50), the quantities $j_{\text {stim }}, \eta_{\text {int }}$ and $P$ can be put in the

$$
\begin{aligned}
j_{\text {stim }} & =j_{\text {capt }} \\
\eta_{\text {int }} & =\frac{j_{\text {capt }}}{j_{\mathrm{OCL}}} \\
P & =\frac{\hbar \omega}{e}\left(S j_{\text {capt }}\right) \eta_{\mathrm{opt}}
\end{aligned}
$$

where $j_{\text {capt }}$ and $j^{\mathrm{OCL}}$ are, respectively, the current densities of the capture into QDs and of the recombination in the OCL at a given injection-current density (not to be confused with their threshold values $j_{\text {capt,th }}$ and $j_{\mathrm{th}}^{\mathrm{OCL}}$ ). The equations for $j^{\mathrm{OCL}}$ and $j_{\text {capt }}$ are (21) and (17), where in place of $n_{\text {th }}$ we now take the carrier density in the OCL above threshold; in (53)-(55), $n$ is given by (50).

Thus, we find that in the limit of high injection currents, the LCC is strongly sublinear (Fig. 2). The photon number, the output power (Fig. 2) and the free-carrier density in the OCL (Fig. 3) all increase as $\sqrt{j-j_{\text {th }}}$, while the internal and external efficiencies decrease as $1 / \sqrt{j-j_{\text {th }}}$ (Fig. 1). These square root dependences are a consequence of the assumed bimolecular $\left(\propto n^{2}\right)$ recombination in the OCL. They can be readily derived from the following simple consideration. At high injection currents, the quadratic (in $j_{\text {stim }}$ ) term in (20) is dominant and we have $j_{\text {stim }} \propto \sqrt{j-j_{\text {th }}}$. Hence, the ratio (30) of the stimulated recombination current (proportional to $j_{\text {stim }}$ ) to the current excess $j-j_{\text {th }}$ (proportional to $j_{\text {stim }}^{2}$ ) becomes inversely proportional to $j_{\text {stim }}$, i.e., $\eta_{\text {int }} \propto 1 / \sqrt{j-j_{\text {th }}}$.

2) Asymptotic Equations for the Case When the Dominant Recombination Channel in the OCL is Auger Recombination: The higher the degree of superlinearity of the recombination rate in the OCL with respect to the carrier density, the higher is the degree of sublinearity of the LCC. Since the nonradiative Auger recombination rate in the OCL increases as $n^{3}$, this recombination channel can become dominant with increasing injection current. In this limit, the difference $j-j_{\text {th }}$ in (29) will be dominated by the cubic term, i.e., $j-j_{\text {th }} \propto j_{\text {stim }}^{3}$. Hence both $j_{\text {stim }}$ and $P$ will be proportional to $\sqrt[3]{j-j_{\text {th }}}$ and the internal quantum efficiency $\eta_{\text {int }}=j_{\text {stim }} /\left(j-j_{\text {th }}\right) \propto$ $j_{\text {stim }} / j_{\text {stim }}^{3}=1 / j_{\text {stim }}^{2} \propto 1 /\left(j-j_{\text {th }}\right)^{2 / 3}$.

Let us derive the corresponding equations. Retaining at high injection-current density only the cubic (in $j_{\text {stim }}$ ) term in (29), we find

$$
j_{\text {stim }}=j_{\text {capt }, \text { th }} \sqrt[3]{\frac{j-j_{\mathrm{th}}}{e b C n_{\mathrm{th}}^{3}}} .
$$

Substituting $j_{\text {stim }}$ from (56) into (14), (30), and (32) gives

$$
\begin{aligned}
n & =\sqrt[3]{\frac{j-j_{\mathrm{th}}}{e b C}} \\
\eta_{\mathrm{int}} & =\frac{j_{\mathrm{capt}, \mathrm{th}}}{\sqrt[3]{e b C n_{\mathrm{th}}^{3}\left(j-j_{\mathrm{th}}\right)^{2}}}=\frac{\sigma_{n} v_{n} N_{S}\left(1-f_{n}\right)}{\sqrt[3]{b C\left(\frac{j-j_{\mathrm{th}}}{e}\right)^{2}}} \\
P & =\frac{\hbar \omega}{e} S j_{\mathrm{capt}, \mathrm{th}} \sqrt[3]{\frac{j-j_{\mathrm{th}}}{e b C n_{\mathrm{th}}^{3}}} \eta_{\mathrm{opt}} \\
& =\hbar \omega\left(N_{S} S\right) \sigma_{n} v_{n}\left(1-f_{n}\right) \sqrt[3]{\frac{j-j_{\mathrm{th}}}{e b C}} \eta_{\mathrm{opt}} .
\end{aligned}
$$

With (58) and (59), the external differential efficiency becomes

$$
\eta_{\mathrm{ext}}=\frac{1}{3} \eta_{\mathrm{int}} \eta_{\mathrm{opt}}
$$

Similar to (52), (60) follows immediately from (36) by taking there $\eta_{\text {int }} \propto 1 /\left(j-j_{\text {th }}\right)^{2 / 3}$.

With (57), the quantities $j_{\text {stim }}, \eta_{\text {int }}$ and $P$ can again be put in the same form (53)-(55), where now $j^{\mathrm{OCL}}$ is given by

$$
j^{\mathrm{OCL}}=e b C n^{3}
$$

and $j_{\text {capt }}$ by (17) with $n$ defined by (57) substituted in place of $n_{\text {th. }}$.

\section{Discussion}

Thus, with increasing current, the linear dependence of the output power on $j-j_{\text {th }}$ changes first to a square-root and then to a cube-root dependence. The higher the excess current $j-j_{\text {th }}$, the larger fraction of it goes into parasitic recombination (first spontaneous and then Auger) outside the active region. When this recombination is the main cause of the LCC sublinearity, the actual shape of the LCC contains valuable information about the dominant recombination channel in the OCL at a given injection-current density.

As seen from (49), (51)-(55), (56) and (58)-(60), at high injection currents, the output characteristics of the laser (the photon number, the internal quantum efficiency and the optical power) are controlled by the carrier capture into QDs [see also the general equations (43) and (44)]. This is particularly apparent from (53)-(55), showing that under high injection (when (48) holds) the stimulated recombination current is purely the capture current. This is also readily seen directly from inequality (48), which describes the condition that the excess of the injection-current density over threshold is much larger than the capture current density at the threshold or, in other words, that the carrier supply into the OCL by injection exceeds the carrier consumption by QDs via the capture process. This means that capture into the active region is the bottleneck and hence carriers accumulate in the OCL much in excess of their threshold amount. This is why the carrier density in the OCL will not depend on the capture cross-section [cf. (50) and (57)] being determined solely by $j-j_{\text {th }}$ and by the specific type of the dominant recombination channel in the OCL. At the same time, the photon number, the power output and the internal and external efficiencies will be strongly limited by the capture cross-section $\sigma_{n}$. This does 
not mean we must necessarily face insurmountable problems associated with carrier capture, such as the phonon bottleneck. As will be shown below, in properly optimized QD laser structures (when parameters are far away from their critical values), the LCC will be linear up to very high injection currents and essentially capture-independent.

Inequality (48) presents a criterion for injection-current densities at which the LCC is strongly sublinear. With other parameters fixed, the larger the capture cross-section, the higher should be $j-j_{\text {th }}$ to enter the nonlinear regime (48). Therefore, structure optimization aimed at enhancing the linearity of LCC would benefit from larger $\sigma_{n}$.

It should be apparent from (14) and (26) that it is the noninstantaneous carrier transfer from the OCL to QDs that causes the free-carrier density in the OCL to increase (Fig. 3). The second term on the right-hand side of these equations, representing the increase in the carrier density above threshold, is capture controlled. As seen from (44), the slower the carrier supply to the active region (the longer $\tau_{\text {capt }}$ ), the larger should be $n-n_{\text {th }}$ (and hence, $j-j_{\text {th }}$ ) to yield a given output power. The free-carrier density would be constant only if carriers could be instantly transferred into the active region [only for $\tau_{\text {capt }}=0$ we get $n=$ $n_{\text {th }}$ in (44)]. This conclusion is general for quantum-confined laser of any dimensionality. When carriers are indirectly supplied to the active region, the carrier density in the feeding reservoir does not pin above threshold (see Fig. 3 and also below). Neither does the spectrum of spontaneous emission from the reservoir (see [16]-[20] and [22] for experimental observation of this in QW lasers); the integrated (over the spectrum) intensity of spontaneous emission increases as $n^{2}-n_{\text {th }}^{2}$.

It is worthwhile to note that the reservoir effect may also have important dynamic manifestations. As is well known, the carrier density unpins when the injection current varies in time and may dip below the threshold value. The finite capture delay, due to the reservoir effect, introduces an additional contribution to this dip, which may be viewed as some kind of a dynamic spatial hole burning [16]. In this paper, however, we consider only the steady-state laser operation, when the carrier density in the active region remains at its threshold value determined by the generation condition.

Another note may be in order here, concerning the assumed linearity (in the carrier density $n$ in the reservoir) of the capture current. Because of this assumption, we obtain a sublinear LCC only if the recombination rate in the reservoir is superlinear. In contrast, when the carrier capture rate itself is nonlinear and grows faster with $n$ than does the recombination rate, then a superlinear LCC is theoretically possible [35].

To summarize, the sublinearity of the LCC at high injection currents is caused by 1) the absence of pinning of the free-carrier density outside the active region (caused in turn by the noninstantaneous carrier capture into the active region) and 2) the superlinearity of the recombination rate with respect to the free-carrier density. This mechanism is inherent to quantum-confined lasers of arbitrary dimensionality. Observations of capture-limited operation of QW lasers have been reported in [17]-[19].

As seen from (53)-(55), in the strongly nonlinear regime (capture-controlled limit) there is no dependence on $n_{\text {th }}$ (except that via $\left.j-j_{\text {th }}\right)$. This is because the free-carrier density in this regime is determined solely by $j-j_{\text {th }}$ [see (50) and (57)].

As seen from (25) and (32), at low injection-current densities and when the structure parameters are well away from their critical values, i.e., when $\eta_{\text {int }}$ is close to unity and the LCC is linear, the dependence of $N$ and $P$ on the structure parameters comes only from the threshold current $I_{\text {th }}$ entering into the difference $I-I_{\text {th }}$; that is to say the structure parameters in this limit only affect the lasing threshold point in the LCC. At high injection-current densities, we see the emergence of a strong dependence of $\eta_{\text {int }}$ and hence of the shape of the light-current curve on the structure parameters (see (49), (51), (52), (56), (58)-(60), and Figs. 1 and 2, as well as Section V for illustrative calculations).

\section{LCC in the Case of High Threshold Current Density}

This case corresponds to large inhomogeneous line broadening, short cavity length (high losses), or small surface density of QDs, when one of the structure parameters is close to its critical tolerable value (maximum QD size dispersion, minimum cavity length, or minimum surface density of QDs). As discussed in Section III-F2, in this case $f_{n} \rightarrow 1$ and the ratio $j_{\text {th }}^{\mathrm{OCL}} / j_{\text {capt,th }} \rightarrow \infty$. In this limit, the quantities $N, n, P$ and $\eta_{\text {ext }}$ are given, respectively, by (25), (26), (32), and (37), with the following expression for $\eta_{\text {int }}$ [obtained from (31)]:

$$
\begin{aligned}
\eta_{\mathrm{int}} & =\frac{j_{\mathrm{capt}, \mathrm{th}}}{j_{\mathrm{th}}^{\mathrm{OCL}}} \frac{1}{1+\sqrt{1+\frac{j-j_{\mathrm{th}}}{j_{\mathrm{th}}}}} \\
& =\frac{\sigma_{n} v_{n} N_{S}\left(1-f_{n}\right)}{b B n_{\mathrm{th}}+\sqrt{\left(b B n_{\mathrm{th}}\right)^{2}+b B \frac{j-j_{\mathrm{th}}}{e}}} .
\end{aligned}
$$

As is evident from (62), in this limit the entire LCC, including its initial portion, is capture-controlled and sublinear (cf. the lowest curve in Fig. 2). This is because the injection-current densities (which must exceed the already high $j_{\text {th }}$ ) are now always in the high $j$ regime, where the laser performance is capture-controlled, as shown above. Note that (49) is immediately obtained from (62) by taking in the latter the limit $j-j_{\text {th }} \gg$ $j_{\mathrm{th}}^{\mathrm{OCL}}$.

\section{ILLUSTRATION OF RESUlTS}

We shall consider a GaInAsP-InP heterostructure similar to that used in previous theoretical studies [26], [29], [30], [32]. Room temperature continuous-wave operation near $1.55 \mu \mathrm{m}$ is assumed. The exemplary device has as-cleaved facets at both ends $(R=0.32)$ and a single layer of QDs (the average size of cubic QDs is $150 \AA$ ). The capture cross-section is plausibly taken to be $\sigma_{n}=10^{-13} \mathrm{~cm}^{-2}$ [26] (which is much less than the geometrical cross-section of a QD).

The optical efficiency is assumed ideal $\eta_{\text {opt }}=1$ (i.e., the internal loss is put $\alpha_{\text {int }}=0$ ). This means that we disregard a possible additional effect on $\eta_{\text {ext }}$ that would result from a freecarrier absorption in the OCL [18], [19].

The root mean square (rms) of relative QD size fluctuations (a Gaussian distribution is assumed), the cavity length and the lateral size are taken to be $\delta=0.05$ (10\% size fluctuations), 


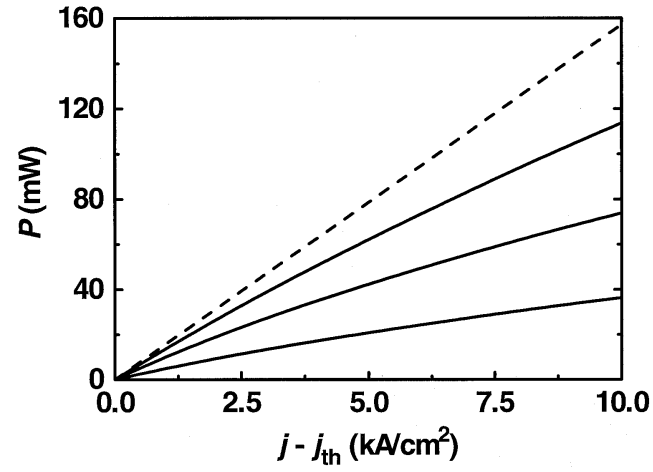

Fig. 6. Light-current characteristics for structures with different rms of relative QD size fluctuation, $\delta$. The dashed line corresponds to the ideal situation, $\eta_{\text {int }}=1$. The values of $\delta$ and $j_{\text {th }}$ (from the top down) are, respectively, $0.13,0.145,0.153$, and $122.44,465.32,1937 \mathrm{~A} / \mathrm{cm}^{2}$.

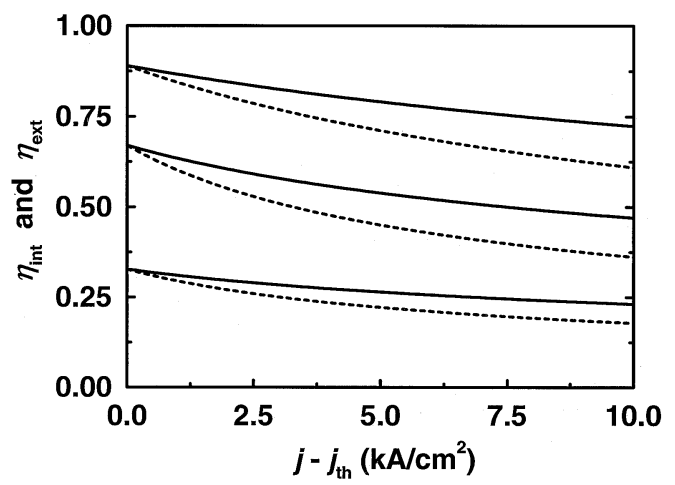

Fig. 7. Injection-current density dependence of the internal quantum efficiency (solid curves) and external differential efficiency (dashed curves) for QD laser structures with different $\delta$ (at fixed cavity length $L$ ) and different $L$ (at fixed $\delta$ ). For $L=1 \mathrm{~mm}$, the values of $\delta$ (from the top down) are 0.13 , 0.145 , and 0.153 . For $\delta=0.05$, the values of $L$ (from the top down) are 385 , 345 , and $327 \mu \mathrm{m}$. The corresponding values of $j_{\text {th }}$ are the same as in Fig. 6.

$L=1 \mathrm{~mm}$ and $W=2 \mu \mathrm{m}$, respectively, unless otherwise specified. For the structure optimized to minimize the threshold current density at above values of $\delta$ and $L$, the surface density of QDs and the OCL thickness are $N_{S}^{\text {opt }}=8.27 \times 10^{10} \mathrm{~cm}^{-2}$ and $b^{\text {opt }}=0.227 \mu \mathrm{m}$. These values of $N_{S}$ and $b$ (used everywhere below, unless otherwise specified) and the minimum threshold current density $j_{\mathrm{th}}^{\min }=12.41 \mathrm{~A} / \mathrm{cm}^{2}$ differ from those calculated in [29]. This is because, to be consistent with our assumption 4) of charge neutrality in both the OCL and the QDs, we have used (19) for $j_{\text {th }}$ (rather than (9) of [29]). The resultant critical tolerable parameters are $N_{S}^{\min }=2.57 \times 10^{10} \mathrm{~cm}^{-2}$, $\delta^{\max }=0.161(32.2 \%)$, and $L^{\min }=311 \mu \mathrm{m}$.

Generally, in an unoptimized device, $\eta_{\text {int }}$ and $\eta_{\text {ext }}$ may deviate widely from unity and the LCC may be highly sublinear. To demonstrate the strong sensitivity of power characteristics on the structure design, we perform calculations at various values of $N_{S}$ (Figs. 1-3), $\delta$ (Figs. 6-8), and $L$ (Fig. 9). As $N_{S}$ decreases below the optimum value, or $\delta$ increases, or $L$ decreases, the output power goes down and the LCC becomes more and more sublinear (Figs. 2, 6, and 9). The internal and external efficiencies depart significantly from unity and their drop with the injection current becomes stronger (Figs. 1 and 7); even at the threshold, they may be several times lower than unity.

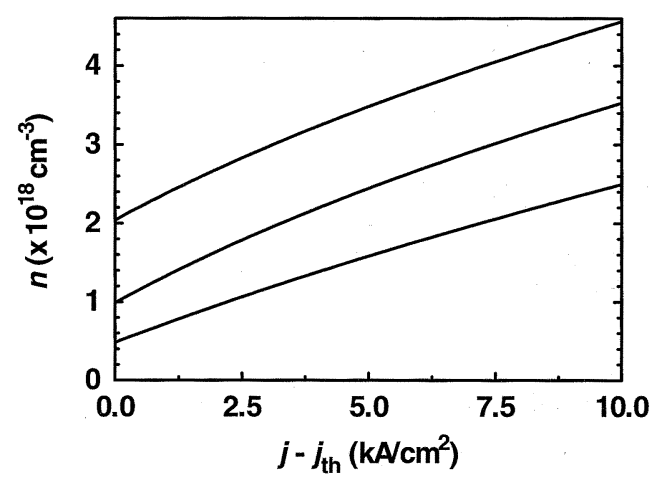

Fig. 8. Injection-current density dependence of the free-carrier density in the OCL for QD laser structures with different $\delta$ (at fixed $L$ ) and various $L$ (at fixed $\delta$ ). The values of $\delta, L$ and $j_{\text {th }}$ (from bottom to top) are the same as in Fig. 7.

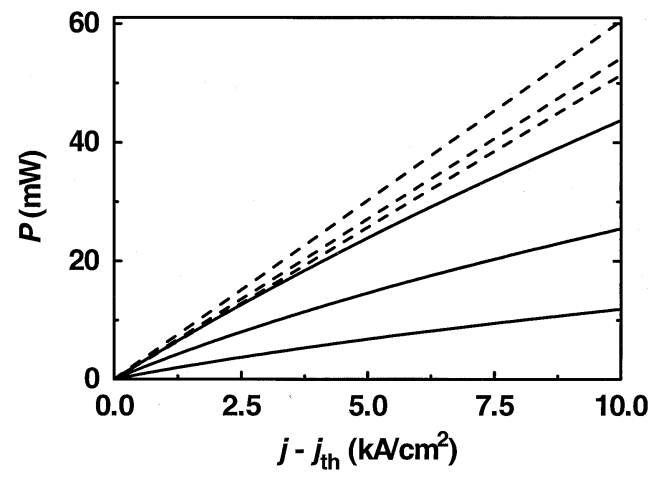

Fig. 9. Light-current characteristics for QD laser structures with different cavity lengths. The dashed lines describe the ideal situation, $\eta_{\text {int }}=1$. The values of $L$ (from the top down) are 385, 345, and $327 \mu \mathrm{m}$. The corresponding values of $j_{\text {th }}$ are the same as in Fig. 6 .

As seen from (26), a relative buildup of the free-carrier density above threshold is

$$
\frac{n-n_{\text {th }}}{n_{\text {th }}}=\frac{j-j_{\text {th }}}{j_{\text {capt }, \text { th }}} \eta_{\text {int }} .
$$

As $f_{n}$ increases (i.e., $N_{S}$ decreases, or $\delta$ increases, or $L$ decreases) at a given $j-j_{\text {th }}$, the internal efficiency $\eta_{\text {int }}$ decreases; simultaneously $j_{\text {capt,th }}$ increases [up to its value at $f_{n}=1$ given by (46)]. Hence, even though the absolute value of $n$ at a given $j-j_{\text {th }}$ grows, the ratio $\left(n-n_{\text {th }}\right) / n_{\text {th }}$ goes down as $f_{n}$ increases. This can be seen from Figs. 3 and 8 .

The threshold quantities $n_{\mathrm{th}}, j_{\mathrm{th}}, j_{\mathrm{th}}^{\mathrm{OCL}}$, and $j_{\mathrm{capt}, \mathrm{th}}$, determining the free-carrier density and the internal and external efficiencies [see (26), (31), and (37)] depend on the rms of QD size fluctuations $\delta$ and the cavity loss $\beta=(1 / L) \ln (1 / R)$ through their product $\delta \beta$. For this reason, Figs. 7 and 8 show $\eta_{\text {int }}, \eta_{\text {ext }}$ and $n$ both at a fixed $L$ but varied $\delta$ and a fixed $\delta$ but varied $L$ - the values of $\delta$ and $L$ are chosen so that $\delta / L$ is the same for both cases.

Since the output power depends on the cavity length not only through $\eta_{\text {int }}$, but also through the trivial dependence on the device area $S=W L$ [see (32)], the asymptotes for the different curves in Fig. 9 are distinct.

For a given injection-current density $j$, Figs. 10-14 illustrate the structure parameter dependences of the photon number, the output power, the quantum efficiencies and the free-carrier density in the OCL. The output power vanishes when $j_{\text {th }}$ is forced 


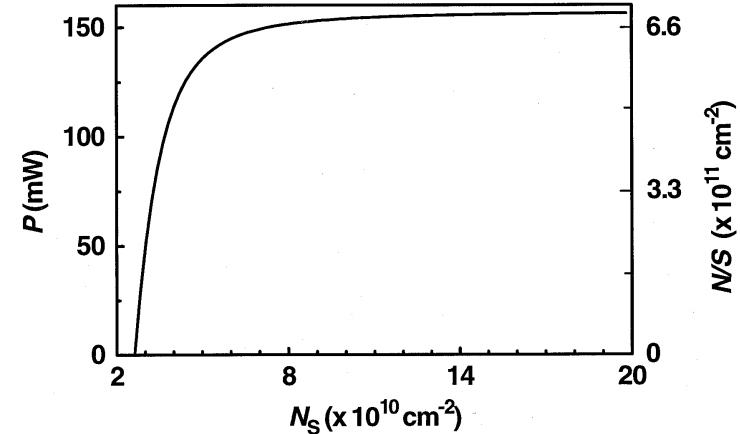

Fig. 10. Output power (left axis) and surface density of photons (right axis) against surface density of QDs. In Figs. 10-14, the injection-current density $j=10 \mathrm{kA} / \mathrm{cm}^{2}$.

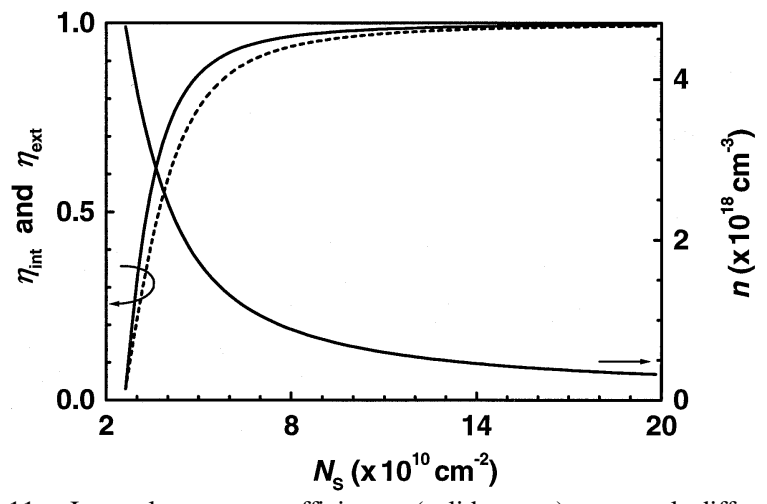

Fig. 11. Internal quantum efficiency (solid curve), external differential efficiency (dashed curve), and free-carrier density in the OCL (right axis) against surface density of QDs.

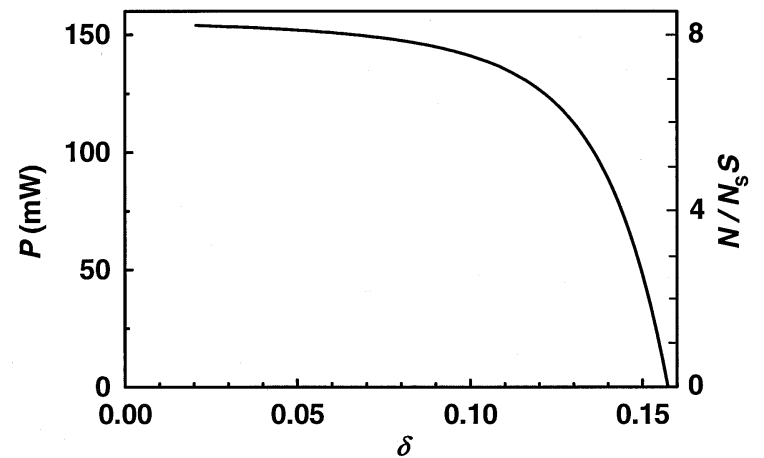

Fig. 12. Output power (left axis) and number of photons per QD (right axis) against rms of relative QD size fluctuations.

to rise to the level of the assumed $j$ (Figs. 10, 12, and 14). We chose the injection-current density so high $\left(j=10 \mathrm{kA} / \mathrm{cm}^{2}\right)$ that this occurs when the structure parameter is near its tolerable limit $\left(v i z ., 1.022 N_{S}^{\min } \approx 2.63 \times 10^{10} \mathrm{~cm}^{-2}, 0.978 \delta^{\max } \approx\right.$ 0.157 , and $1.022 L^{\min }=318 \mu \mathrm{m}$ in Figs. 10, 12, and 14, respectively), i.e., when the QD level occupancy is close to unity $\left(f_{n} \approx 0.989\right)$. As discussed above [see (62)], both $\eta_{\text {int }}$ and $\eta_{\text {ext }}$ vanish in this limit (Figs. 11 and 13).

Conversely, as the structure parameter moves away from its critical tolerable value $\left(N_{S}\right.$ increases, or $\delta$ decreases, or $L$ increases), the surface density of photons (25) increases. As the level occupancy in a QD tends to $1 / 2$, the photon density saturates. In this limit $N / S$ is independent of the structure parameters (Figs. 10, 12, and 14), being determined solely by the excess of $j$ over $j_{\text {th }}$. Both the internal and external efficiencies increase and saturate at unity (Figs. 11 and 13). The

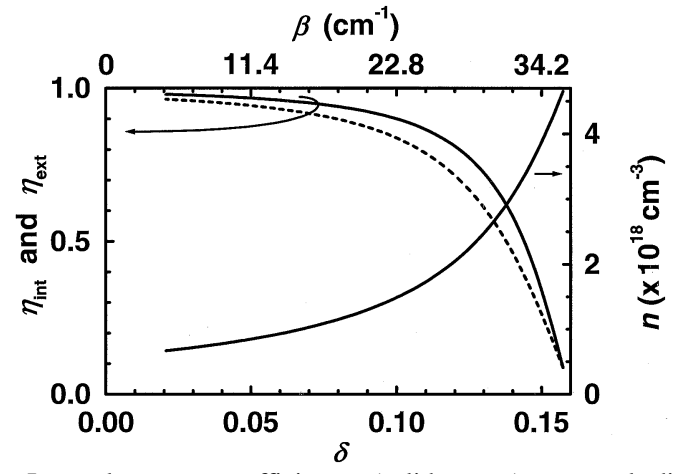

Fig. 13. Internal quantum efficiency (solid curve), external differential efficiency (dashed curve) and free-carrier density in the OCL (right axis) against rms of relative QD size fluctuations (at fixed $L=1 \mathrm{~mm}$, bottom axis) and against cavity losses (at fixed $\delta=0.05$, top axis).

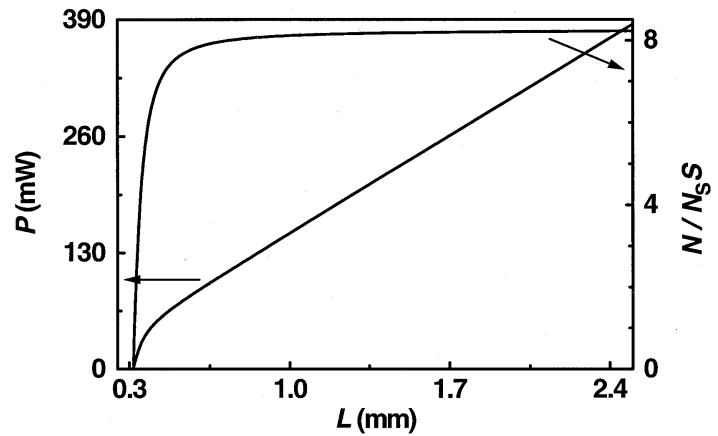

Fig. 14. Output power (left axis) and number of photons per QD (right axis) against cavity length.

output power also saturates with increasing $N_{S}$ or decreasing $\delta$ (Figs. 10 and 12). As $L$ increases, the output power becomes linear in $L$, following the apparent dependence on the device area $S=W L$ (Fig. 14). With the parameter moving away from its critical tolerable value, the free-carrier density drops significantly (Figs. 11 and 13). Note that the dependences on both $\delta$ and $L^{-1}$ are shown simultaneously in Fig. 13, since the product $\delta \beta[\beta=(1 / L) \ln (1 / R)]$ is varied over the same range in both the bottom and top axes (see the earlier comment on Figs. 7 and $8)$.

Thus, Figs. 10-14 clearly demonstrate the crucial importance of properly controlling the structure parameters in a QD laser intended for high-power applications. This primarily refers to the QD size dispersion and the surface density of QDs, since varying the cavity length is not much of a problem.

For the optimized structure, the level occupancy in a QD is far from unity $\left(f_{n}=0.655\right)$ and the threshold current density is very low $\left(j_{\mathrm{th}}^{\min }=12.41\right)$. As follows from the above analysis, in this case $\eta_{\text {int }}$ should be close to unity and the LCC should be linear. Figs. 15 and 16 show the photon number, the free-carrier density in the OCL, the output power and the internal and external quantum efficiencies against $j-j_{\text {th }}$ (the bottom axis) and $I-I_{\mathrm{th}}$ (the top axis) for an optimized broad-area device [with the lateral size (the stripe width) $W=100 \mu \mathrm{m}$ ]. Notably, the photon number per QD increases from 0 to about 12 in the range of the injection-current density shown (Fig. 15). The carrier density in the OCL (Fig. 16) increases by more than an order of magnitude (from $9.7 \times 10^{16}$ to $1.21 \times 10^{18} \mathrm{~cm}^{-3}$ ). As Fig. 15 suggests, for optimized structures (at least when the parameters are well away from their critical tolerable values), the LCC is 


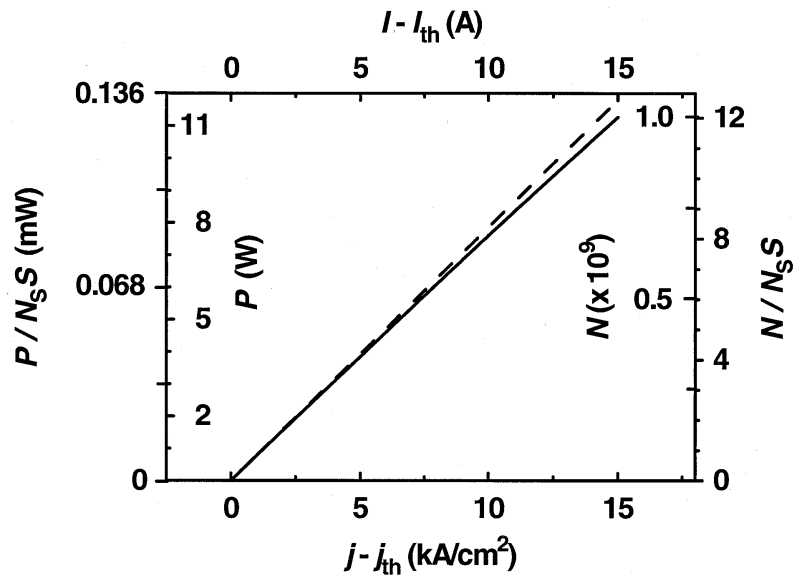

Fig. 15. Injection-current density dependence of the output power (left axis) and photon number (right axis) for an optimized broad-area QD laser structure $\left(N_{S}^{\text {opt }}=8.268 \times 10^{10} \mathrm{~cm}^{-2}, b^{\text {opt }}=0.227 \mu \mathrm{m}, j_{\text {th }}=12.41 \mathrm{~A} / \mathrm{cm}^{2}\right.$ and $W=100 \mu \mathrm{m})$. The dashed line corresponds to the ideal situation, $\eta_{\text {int }}=1$. Power and photon number per QD are also shown. The top axis shows the excess injection current.

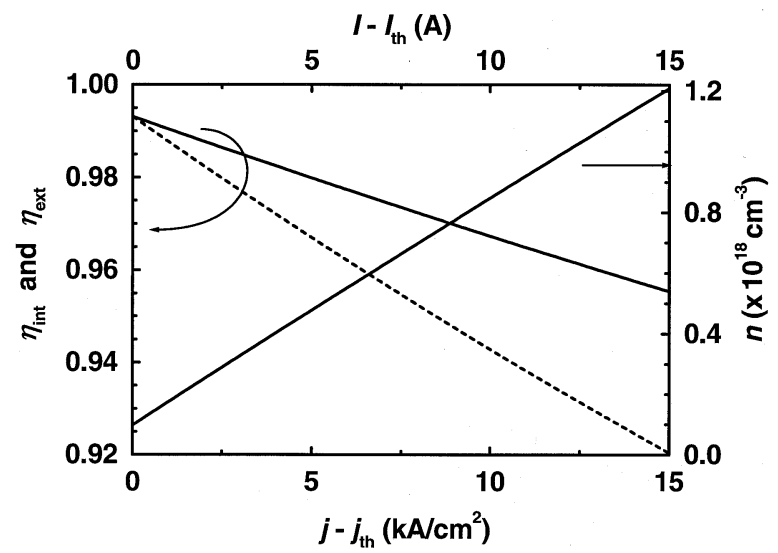

Fig. 16. Injection-current density dependence of internal quantum efficiency (solid curve), external differential efficiency (dashed curve) and free-carrier density in the OCL (right axis) for an optimized QD laser structure as in Fig. 15. The top axis shows the excess injection current.

practically linear up to very high $j$. The internal and external efficiencies, being close to unity, decrease by only $3.8 \%$ and $7.3 \%$, respectively, as $j$ increases by more than three orders of magnitude (from $12.41 \mathrm{~A} / \mathrm{cm}^{2}$ to $15 \mathrm{kA} / \mathrm{cm}^{2}$ ) (Fig. 16). Hence, the feasibility of properly designed QD lasers for high-power generation is theoretically justified here. This is best demonstrated by Fig. 15-the output power exceeds $11 \mathrm{~W}$ at $j=15 \mathrm{kA} / \mathrm{cm}^{2}$ ( $I=15 \mathrm{~A}$ ); for this current, $\eta_{\text {int }}=0.955$ and $\eta_{\text {ext }}=0.92$.

\section{Generalization to Semiconductor Lasers With A QUANTUM-CONFINED ACTIVE REgION OF ARBITRARY DIMENSIONALITY}

In this section, we show that (31) and (37) for $\eta_{\text {int }}$ and $\eta_{\text {ext }}$ obtained for a QD laser, remain valid also for QW and QWR lasers, supplemented by modified equations for $j_{\text {capt,th }}$ and $j_{\mathrm{th}}^{\mathrm{OCL}}$.

In the general case, the steady-state rate equations for the carriers confined in the active region, the free carriers in the OCL and the photons can be written as follows:

$$
\begin{array}{r}
j_{\text {capt }}-j_{\text {esc }}-j_{\text {spon }}^{\text {active }}-j_{\text {stim }}=0 \\
j_{\text {esc }}-j_{\text {capt }}-j^{\text {OCL }}+j=0
\end{array}
$$

$$
j_{\mathrm{stim}}-e \frac{1}{S} \frac{N}{\tau_{\mathrm{ph}}}=0
$$

where $j_{\text {capt }}, j_{\text {esc }}, j_{\text {stim }}$, and $j^{\mathrm{OCL}}$ are the current densities associated with the same processes as in the case of a QD laser and $j_{\text {spon }}^{\text {active }}$ is the spontaneous radiative recombination current density in the active region; $j_{\text {stim }}=(e / S)\left(c / \sqrt{\epsilon_{g}}\right) g N$ with $g$ being the modal gain spectrum peak in the active region.

The capture current density is

$$
j_{\text {capt }}=\frac{e b n}{\tau_{\text {capt }}}=\frac{e b n\left(1-f_{n}\right)}{\tau_{\text {capt }, 0}}
$$

where [cf. (41)] $\tau_{\text {capt }, 0}$ is the "capture time" into an empty quantum-confined active region (when the subband-edge level occupancy $f_{n}=0$ ).

Equation (67) can be put in the equivalent form

$$
j_{\text {capt }}=e n v_{\text {capt }}=e n\left(1-f_{n}\right) v_{\text {capt }, 0}
$$

where we have formally introduced the capture velocity (in centimeters per second) by

$$
v_{\text {capt }}=\frac{b}{\tau_{\text {capt }}} .
$$

The velocity of capture into an empty quantum-confined active region is denoted by $v_{\text {capt }, 0}$ so that $v_{\text {capt }}=\left(1-f_{n}\right) v_{\text {capt }, 0}$. The capture velocity is an analog of the surface recombination velocity.

For a $\mathrm{QW}$, it is $v_{\text {capt, } 0}$ that describes adequately the capture process and that should be calculated from the first principles.

With (41), we get for a QD active region (with $N_{S}$ being the surface density of QDs)

$$
v_{\text {capt }, 0}=\sigma_{n} v_{n} N_{S} .
$$

For a specific structure considered in Section V, $v_{\text {capt }, 0}=2.3 \times$ $10^{5} \mathrm{~cm} / \mathrm{s}$. To express $j_{\text {capt }}$ in terms of the "capture time" [see (67)], an "artificial" dependence of the latter on the OCL thickness $b$ has been introduced in a QD case [see (41)]. This dependence drops out of (68). In contrast to $\tau_{\text {capt }, 0}$, there is no such artificial dependence in $v_{\text {capt }, 0}$. Hence, $v_{\text {capt }, 0}$ is a "better" parameter than $\tau_{\text {capt }, 0}$. However, it still does not adequately describe the capture process into a single QD. Like $\tau_{\text {capt }, 0}$, the constant $v_{\text {capt }, 0}$ is a characteristic of the entire QD ensemble. An adequate physical quantity, describing correctly the carrier capture into a single $\mathrm{QD}$, is the capture cross-section $\sigma_{n}$, cf. Section III-F1.

Similarly, for a QWR active region we define

$$
v_{\text {capt }, 0}=l_{n} v_{n} N_{L}
$$

where $N_{L}$ is the linear density of QWRs (in $\mathrm{cm}^{-1}$ ) and $l_{n}$ is the cross-length of the carrier capture into a QWR. In view of the (formally) infinite length of a QWR along the direction of free motion of carriers, it is the capture cross-length, rather than the cross-section, that is the appropriate physical quantity to describe the capture into a QWR (in contrast to the QD case, where the capture process is adequately described by a cross-section $\left.\sigma_{n}\right)$.

The current density of the carrier escape from QWs is

$$
j_{\mathrm{esc}}=e N_{\mathrm{QW}} \frac{n_{2 \mathrm{D}}}{\tau_{\mathrm{esc}}}
$$


where $N_{\mathrm{QW}}$ is the number of QWs, $n_{2 \mathrm{D}}$ is the 2-D carrier density in a $\mathrm{QW}$ (in $\mathrm{cm}^{-2}$ ). For a QD laser, $j_{\mathrm{esc}}$ is given by (23). For a QWR laser, we have

$$
j_{\mathrm{esc}}=e N_{L} \frac{n_{1 \mathrm{D}}}{\tau_{\mathrm{esc}}}
$$

where $n_{1 \mathrm{D}}$ is the 1-D carrier density in a QWR $\left(\right.$ in $\left.\mathrm{cm}^{-1}\right)$.

The spontaneous radiative recombination current density in QWs is

$$
j_{\mathrm{spon}}^{\text {active }}=e N_{\mathrm{QW}} B_{2 \mathrm{D}} n_{2 \mathrm{D}}^{2}
$$

where $B_{2 \mathrm{D}}$ is the radiative constant for a 2-D region (in $\mathrm{cm}^{2} / \mathrm{s}$ ). The analogous quantity for QDs is given by (24). For QWRs

$$
j_{\text {spon }}^{\text {active }}=e N_{L} B_{1 \mathrm{D}} n_{1 \mathrm{D}}^{2}
$$

where $B_{1 \mathrm{D}}$ is the radiative constant for a 1 -D region (in centimeters per second).

When spontaneous radiation is the dominant recombination channel in the OCL, then

$$
j^{\mathrm{OCL}}=e b B n^{2}
$$

where $B$ is the radiative constant for bulk (3-D) region (in $\mathrm{cm}^{3} / \mathrm{s}$ ) and $n$ is the free-carrier density in the OCL.

When the nonradiative Auger recombination dominates, then $j^{\mathrm{OCL}}$ is given by (61).

From (66) follows (16) for $j_{\text {stim }}$ and we immediately conclude that the carrier density in the active region pins above threshold. Both $j_{\mathrm{esc}}$ and $j_{\mathrm{spon}}^{\text {active }}$, since they are controlled by the carrier density in the active region [see (72)-(75)], clamp above threshold; hence, using (64), we regain (14) [or equivalent (26)] for the free-carrier density outside the active region. In this equation, the free-carrier density at the lasing threshold is of the form

$$
n_{\mathrm{th}}=\frac{j_{\mathrm{capt}, \mathrm{th}}}{e v_{\mathrm{capt}}}=\frac{j_{\mathrm{esc}}+j_{\mathrm{spon}}^{\mathrm{active}}}{e v_{\mathrm{capt}}}=\frac{j_{\mathrm{esc}}+j_{\mathrm{spon}}^{\mathrm{active}}}{e\left(1-f_{n}\right) v_{\mathrm{capt}, 0}} .
$$

For a QD laser, $n_{\text {th }}$ is given by (15). For a QW laser, using (72) and (74), we have

$$
n_{\text {th }}=N_{\mathrm{QW}} \frac{1}{v_{\text {capt }, 0} \tau_{\text {esc }}} \frac{n_{2 \mathrm{D}}}{1-f_{n}}+N_{\mathrm{QW}} \frac{B_{2 \mathrm{D}}}{v_{\text {capt }, 0}} \frac{n_{2 \mathrm{D}}^{2}}{1-f_{n}} .
$$

Note that since $v_{\text {capt }, 0}$ is proportional to the number $N_{\mathrm{QW}}$ of QWs, $N_{\mathrm{QW}}$ drops out of (78).

For a QWR laser, using (73), (75) and (71), we find

$$
n_{\mathrm{th}}=\frac{1}{l_{n} v_{n} \tau_{\text {esc }}} \frac{n_{1 \mathrm{D}}}{1-f_{n}}+\frac{B_{1 \mathrm{D}}}{l_{n} v_{n}} \frac{n_{1 \mathrm{D}}^{2}}{1-f_{n}} .
$$

With (64) and (65), the injection-current density is expressed as

$$
j=j^{\mathrm{OCL}}+j_{\mathrm{spon}}^{\text {active }}+j_{\mathrm{stim}} .
$$

Since $j_{\mathrm{spon}}^{\text {active }}$ pins above threshold, the excess injection-current density $j-j_{\text {th }}$ becomes

$$
j-j_{\mathrm{th}}=j^{\mathrm{OCL}}-j_{\mathrm{th}}^{\mathrm{OCL}}+j_{\mathrm{stim}}
$$

where

$$
j_{\mathrm{th}}=j_{\mathrm{th}}^{\mathrm{OCL}}+j_{\mathrm{spon}}^{\text {active }}
$$

with $j_{\mathrm{th}}^{\mathrm{OCL}}$ being the value of $j^{\mathrm{OCL}}$ at $n=n_{\mathrm{th}}$. For a QD laser, $j_{\text {th }}$ is given by (19); analogous expressions for QW and QWR lasers are readily obtained from (82), (76), (74), (75), (78), and (79).

Substituting in (81) the expression for $j^{\mathrm{OCL}}$ from (76) [with $n$ given by (14)] yields (20) for $j_{\text {stim }}$.

When the nonradiative Auger recombination in the OCL is also included, the previous equation (29) is regained for $j_{\text {stim }}$.

Thus, all our earlier results and conclusions [including (31) and (37) for $\eta_{\text {int }}$ and $\eta_{\text {ext }}$ ] derived from (20) for a QD laser, remain valid for lasers with a quantum-confined active region of an arbitrary dimensionality.

In particular, this applies to our conclusion about critical dependences on the structure design. Thus, from (77)-(79) it follows that $n_{\text {th }} \rightarrow \infty$ as $f_{n} \rightarrow 1$. For QW lasers, the critical tolerable parameters are the minimum number of QWs and the minimum cavity length. For QWR lasers, these are the minimum linear density of QWRs, the maximum QWR size dispersion and the minimum cavity length.

\section{CONCLUSIONS}

Carrier density in $\mathrm{QD}, \mathrm{QWR}$, and QW lasers pins above threshold only in the active region. In regions abutting and feeding carriers by a capture process to the quantum-confined region, the carrier density rises with injection current, owing to a nonvanishing capture delay. This "reservoir effect", combined with the superlinearity of the recombination rate with respect to the carrier density outside the active region, gives rise to a new type of sublinearity of the LCC at high injection and limits the power performance of semiconductor lasers with a quantum-confined active region.

We have developed a theory of this effect under steady-state conditions and derived for the first time a universal closed-form expression for the current dependence of the internal differential quantum efficiency $\eta_{\text {int }}$. In this dependence, the key parameter is the dimensionless ratio of the recombination current in the reservoir to carrier capture current, both taken at threshold. The universal expression retains the same form for QD, QWR, and QW lasers.

The actual shape of nonlinear LCC depends on the dominant recombination channel outside the active region. For spontaneous radiative bimolecular $\left(\propto n^{2}\right)$ recombination or nonradiative Auger $\left(\propto n^{3}\right)$ recombination in the OCL, the output power at high injection currents increases as $\sqrt{j-j_{\text {th }}}$ or $\sqrt[3]{j-j_{\text {th }}}$, respectively, and the quantum efficiency decreases as $\left(j-j_{\mathrm{th}}\right)^{-1 / 2}$ or $\left(j-j_{\mathrm{th}}\right)^{-2 / 3}$, respectively. Analysis of the LCC shape provides, therefore, a method for identifying the dominant recombination channel in the OCL.

We demonstrate a direct relationship between the power and the threshold characteristics in the sense that reducing the threshold current density is a key to increasing the output power and internal quantum efficiency. This indicates that for high-power applications, QD lasers may have a major advantage over conventional QW lasers.

Our general results have been illustrated in detail in the instance of QD lasers and their high-power performance. This analysis should be highly relevant in the design of such lasers, 
since we show that the power performance of a QD laser critically depends on such structure parameters as the surface density of QDs, the QD-size dispersion and the cavity length. When one of the structure parameters $\left(N_{S}, \delta\right.$, or $\left.L\right)$ approaches its critical tolerable value $\left(N_{S}^{\min }, \delta^{\max }\right.$, or $L^{\mathrm{min}}$, respectively) and hence the threshold current density tends to infinity, both the output power and the quantum efficiency vanish. As this takes place, the LCC is initially sublinear, being always controlled by the capture process. On the other hand, as the structure parameter is moved away from its critical value, the structural dependence of $n, N, P / L, \eta_{\text {int }}$ and $\eta_{\text {ext }}$ reduces to the dependence of the threshold current density on $N_{S}, \delta$ and $L$, i.e., to the structural dependence of the threshold point.

In properly optimized QD lasers, when the structure parameters are far away from their critical tolerable values, the LCC is linear with both the internal quantum efficiency and the external differential efficiency being close to unity up to very high injection-current densities $\left(15 \mathrm{kA} / \mathrm{cm}^{2}\right)$. Output powers in excess of $10 \mathrm{~W}$ at the internal quantum efficiency higher than $95 \%$ have been shown to be attainable in broad-area QD devices.

We note, finally, a radically new design strategy, recently proposed to improve the temperature stability of QD lasers [33], [34]. In this approach, the two reservoirs feeding carriers into the quantum confined region are essentially unipolar and the finite-delay capture process is not accompanied by a build-up of a bipolar carrier density and additional recombination. We therefore expect that lasers designed according to [33], [34] will exhibit linear behavior and excellent power performance.

\section{REFERENCES}

[1] R. Dingle and C. H. Henry, "Quantum Effects in Heterostructure Lasers," U.S. Patent 3982 207, Sept. 21, 1976.

[2] W. T. Tsang, "Extremely low threshold (AlGa)As graded-index waveguide separate-confinement heterostructure lasers grown by molecular-beam epitaxy," Appl. Phys. Lett., vol. 40, no. 3, pp. 217-219, 1982.

[3] P. S. Zory Jr., Ed., Quantum Well Lasers. Boston, MA: Academic, 1993, p. 504.

[4] Y. Arakawa and H. Sakaki, "Multidimensional quantum well laser and temperature dependence of its threshold current," Appl. Phys. Lett., vol. 40, no. 11, pp. 939-941, June 1982.

[5] N. Kirstädter, N. N. Ledentsov, M. Grundmann, D. Bimberg, V. M. Ustinov, S. S. Ruvimov, M. V. Maximov, P. S. Kop'ev, Z. I. Alferov, U. Richter, P. Werner, U. Gösele, and J. Heydenreich, "Low threshold, large $T_{0}$ injection laser emission from (InGa)As quantum dots," Electron. Lett., vol. 30, no. 17, pp. 1416-1417, Aug. 1994.

[6] R. Mirin, A. Gossard, and J. Bowers, "Room temperature lasing from InGaAs quantum dots," Electron. Lett., vol. 32, no. 18, pp. 1732-1734, Aug. 1996.

[7] A. R. Kovsh, A. E. Zhukov, D. A. Lifshits, A. Y. Egorov, V. M. Ustinov, M. V. Maximov, Y. G. Musikhin, N. N. Ledentsov, P. S. Kop'ev, Z. I. Alferov, and D. Bimberg, "3.5 W CW operation of quantum dot laser," Electron. Lett., vol. 35, no. 14, pp. 1161-1163, July 1999.

[8] T. C. Newell, D. J. Bossert, A. Stintz, B. Fuchs, K. J. Malloy, and L. F. Lester, "Gain and linewidth enhancement factor in InAs quantum-dot laser diodes," IEEE Photon. Technol. Lett., vol. 11, pp. 1527-1529, Dec. 1999.

[9] P. G. Eliseev, H. Li, G. T. Liu, A. Stintz, T. C. Newell, L. F. Lester, and K. J. Malloy, "Ground-state emission and gain in ultralow-threshold InAsInGaAs quantum-dot lasers," IEEE J. Select. Topics Quantum Electron., vol. 7, pp. 135-142, Mar./Apr. 2001.

[10] N. N. Ledentsov, M. Grundmann, F. Heinrichsdorff, D. Bimberg, V. M. Ustinov, A. E. Zhukov, M. V. Maximov, Z. I. Alferov, and J. A. Lott, "Quantum-dot heterostructure lasers," IEEE J. Select. Topics Quantum Electron., vol. 6, pp. 439-451, May/June 2000.
[11] P. Bhattacharya, D. Klotzkin, O. Qasaimeh, W. Zhou, S. Krishna, and D. Zhu, "High-speed modulation and switching characteristics of $\mathrm{In}(\mathrm{Ga}) \mathrm{As}-\mathrm{Al}(\mathrm{Ga})$ as self-organized quantum-dot lasers," IEEE J. Select. Topics Quantum Electron., vol. 6, pp. 426-438, May/June 2000.

[12] G. Park, O. B. Shchekin, D. L. Huffaker, and D. G. Deppe, "Low-threshold oxide-confined $1.3-\mu \mathrm{m}$ quantum-dot laser," IEEE Photon. Technol. Lett., vol. 13, pp. 230-232, Mar. 2000.

[13] M. Sugawara, K. Mukai, Y. Nakata, K. Otsubo, and H. Ishilkawa, "Performance and physics of quantum-dot lasers with self-assembled columnar-shaped and 1.3- $\mu \mathrm{m}$ emitting InGaAs quantum dots," IEEE J. Select. Topics Quantum Electron., vol. 6, pp. 462-474, May/June 2000.

[14] F. Klopf, J. P. Reithmaier, A. Forchel, P. Collot, M. Krakowski, and M. Calligaro, "High-performance $980 \mathrm{~nm}$ quantum dot lasers for highpower applications," Electron. Lett., vol. 37, pp. 353-354, Mar. 2001.

[15] P. M. Smowton, E. Herrmann, Y. Ning, H. D. Summers, P. Blood, and M. Hopkinson, "Optical mode loss and gain of multiple-layer quantum-dot lasers," Appl. Phys. Lett., vol. 78, no. 18, pp. 2629-2631, Apr. 2001.

[16] W. Rideout, W. F. Sharfin, E. S. Koteles, M. O. Vassell, and B. Elman, "Well-barrier hole burning in quantum-well lasers," IEEE Photon. Technol. Lett., vol. 3, pp. 784-786, Sept. 1991.

[17] N. Tessler, R. Nagar, G. Eisenstein, S. Chandrasekhar, C. H. Joyner, A. G. Dentai, U. Koren, and G. Raybon, "Nonequilibrium effects in quantum well lasers," Appl. Phys. Lett., vol. 61, no. 20, pp. 2383-2385, Nov. 1992.

[18] H. Hirayama, J. Yoshida, Y. Miyake, and M. Asada, "Estimation of carrier capture time of quantum-well lasers by spontaneous emission spectra," Appl. Phys. Lett., vol. 61, no. 20, pp. 2398-2400, Nov. 1992.

[19] - "Carrier capture time and its effect on the efficiency of quantum-well lasers," IEEE J. Quantum Electron., vol. 30, pp. 54-62, Jan. 1994.

[20] L. A. Coldren and S. W. Corzine, Diode Lasers and Photonic Integrated Circuits. New York: Wiley, 1995, p. 594.

[21] G. W. Taylor and P. R. Claisse, "Transport solutions for the SCH quantum-well laser-diode," IEEE J. Quantum Electron., vol. 31, pp. 2133-2141, Dec. 1995.

[22] P. M. Smowton and P. Blood, "The differential efficiency of quantum-well lasers," IEEE J. Select. Topics Quantum Electron., vol. 3, pp. 491-498, Apr. 1997.

[23] G. W. Taylor and S. Jin, "Revisions to transport solution for SCH QW laser diodes," IEEE J. Quantum Electron., vol. 34, pp. 1886-1889, Oct. 1998.

[24] D. Botez, "Design considerations and analytical approximations for high continuous-wave power, broad-waveguide diode lasers," Appl. Phys. Lett., vol. 74, no. 21, pp. 3102-3104, May 1999.

[25] A. Al-Muhanna, L. J. Mawst, D. Botez, D. Z. Garbuzov, R. U. Martinelli, and J. C. Connolly, "High-power $(>10 \mathrm{~W}$ ) continuous-wave operation from $100-\mu \mathrm{m}$-aperture $0.97-\mu \mathrm{m}$-emitting Al-free diode lasers," Appl. Phys. Lett., vol. 73, no. 9, pp. 1182-1184, Aug. 1998.

[26] L. V. Asryan and R. A. Suris, "Longitudinal spatial hole burning in a quantum-dot laser," IEEE J. Quantum Electron., vol. 36, pp. 1151-1160, Oct. 2000.

[27] R. Nagarajan, M. Ishikawa, T. Fukushima, R. S. Geels, and J. E. Bowers, "High speed quantum-well lasers and carrier transport effects," IEEE J. Quantum Electron., vol. 28, pp. 1990-2008, Oct. 1992.

[28] M. Ishikawa, R. Nagarajan, T. Fukushima, J. G. Wasserbauer, and J. E. Bowers, "Long wavelength high-speed semiconductor-lasers with carrier transport effects," IEEE J. Quantum Electron., vol. 28, pp. 2230-2241, Oct. 1992.

[29] L. V. Asryan and R. A. Suris, "Inhomogeneous line broadening and the threshold current density of a semiconductor quantum dot laser," Semicond. Sci. Technol., vol. 11, pp. 554-567, Apr. 1996.

[30] — - "Charge neutrality violation in quantum dot lasers," IEEE J. Select. Topics Quantum Electron., vol. 3, pp. 148-157, Apr. 1997.

[31] G. P. Agrawal and N. K. Dutta, Long-Wavelength Semiconductor Lasers. New York: Van-Nostrand Reinhold, 1986, p. 474.

[32] L. V. Asryan and R. A. Suris, "Temperature dependence of the threshold current density of a quantum dot laser," IEEE J. Quantum Electron., vol. 34, pp. 841-850, May 1998.

[33] L. V. Asryan and S. Luryi, "Tunneling-injection quantum-dot laser: Ultrahigh temperature stability," IEEE J. Quantum Electron., vol. 37, pp. 905-910, July 2001.

[34] - "Temperature-insensitive semiconductor quantum dot laser," Solid-State Electron., vol. 47, no. 2, pp. 205-212, Feb. 2003.

[35] A. V. Uskov, Y. Boucher, J. L. Bihan, and J. McInerney, "Theory of a self-assembled quantum-dot semiconductor laser with auger carrier capture: Quantum efficiency and nonlinear gain," Appl. Phys. Lett., vol. 73, no. 11, pp. 1499-1501, Sept. 1998. 


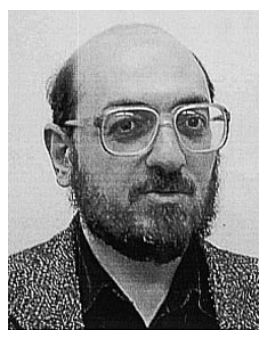

Levon V. Asryan was born in Talin, Armenia, in 1963. He received the M.Sc. degree in radiophysics and electronics from the Yerevan State University, Armenia, in 1985, the Ph.D. degree in physics and mathematics in 1988, and the Doctor of Physical and Mathematical Sciences degree in 2002, both from the Ioffe Physico-Technical Institute, St. Petersburg, Russia.

Since 1992, he has been a Member of Scientific Staff at the Ioffe Institute (since 1999 as a Senior Member). He is currently a Research Associate Professor in the Department of Electrical and Computer Engineering, State University of New York at Stony Brook, on leave from the Ioffe Institute. His research interests include the physics of semiconductors and semiconductor devices, nanoelectronics, optoelectronics, and photonics. He has developed, in collaboration with R. A. Suris, a theory of threshold characteristics of quantum-dot lasers. During 1995-1998, he was involved in the simulation of threshold and highpower characteristics of multiple-quantum-well lasers for Nortel Networks.

Dr. Asryan was a co-recipient of the State Prize of the Russian Federation (the highest Russian Scientific award) for the year 2001 for theoretical work on quantum-dot lasers.

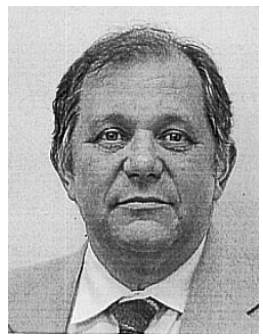

Serge Luryi (M'81-SM'85-F'90) received the Ph.D. degree in physics from the University of Toronto, ON, Canada, in 1978. His doctoral thesis was devoted to quantum mechanics of intermolecular interactions in solid hydrogen.

In 1980, he joined Bell Laboratories, Murray Hill, NJ, and became interested in the physics and technology of semiconductor devices. In 1994, he joined the University at Stony Brook, Stony Brook, NY, where he is currently a Leading Professor and Chair of Electrical and Computer Engineering. He is also Director of the NY State Center for Advanced Sensor Technology. He has published over 180 scientific papers and holds 34 U.S. Patents. In 1995, he organized an advanced research workshop on the "Future Trends in Microelectronics: Reflections on the Road to Nanotechnology", which grew into a regular series. The fourth workshop in this series, "The Nano, the Giga, the Ultra and the Bio", is scheduled for June 2003 on the island of Corsica.

Dr. Luryi served as the Editor of IEEE TRANSACTIONS ON ELECTRON DEVICES during 1986-1990. He was elected Fellow of the IEEE for contributions in the field of heterojunction devices in 1989, received the Distinguished Member of Technical Staff award from Bell Laboratories in 1990, and was elected Fellow of the American Physical Society in 1993, for contributions to the theory of electron transport in low-dimensional systems and invention of novel electron devices.

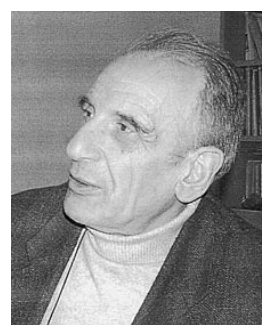

Robert A. Suris graduated from the Moscow Institute of Steel and Alloys, Moscow, Russia, in 1960. He received the Ph.D. degree in solid state theory from the Moscow State University in 1964 and the Doctor of Physical and Mathematical Sciences degree from the Institute of Radioengineering, Moscow, in 1974.

From 1964 to 1988, he was a Member of Scientific Staff at the Microelectronics Research Center, Zelenograd, Russia. Concurrently, during 1970-1988, he served as a Professor in the Department of Physics and Quantum Electronics, Moscow Physico-Technical Institute. In 1988, he joined the Scientific Staff of the Ioffe Physico-Technical Institute, St. Petersburg, Russia, as the Head of the Department of Theoretical Bases of Microelectronics. During 1993-1999, he was also the Director of the Division of Solid State Electronics at the Ioffe Institute. Concurrently, he is the Chair of Solid State Physics in the Department of Physics and Technology, St. Petersburg State Technical University, St. Petersburg, Russia. His research interests include the theory of semiconductors and semiconductor nanostructures, theory of solid-state devices, microelectronics and optoelectronics technology, statistical mechanics, and phase transitions. He has published over 270 papers on the theory of electric, photoelectric, and optical phenomena in semiconductors and semiconductor devices, physics of semiconductor lasers, semiconductor structures characterization, theory of microlithography, theory of phase transitions, diffusion-controlled kinetics, and epitaxial growth. He holds over 20 patents in semiconductor technology.

Dr. Suris is a Member of the Editorial Boards of Technical Physics, Technical Physics Letters (St. Petersburg), and Microelectronics (Moscow). During 1995-1998, he was a Member of the Editorial Board of Semiconductor Science and Technology, IOPP, U.K. He served as a Program Committee Member for a number of Russian and International conferences. He is the permanent Program Chair of the Annual International Symposium "Nanostructures: Physics and Technology" (1993-present), held in St. Petersburg, Russia. In 1997, he was elected a Corresponding Member of the Russian Academy of Sciences. In 1998, he was a co-recipient of the Rank Prize for optoelectronics for the invention of quantum-cascade lasers. In 2001, he was awarded the State Prize of the Russian Federation for theoretical work on quantum-dot lasers. 